\title{
ON THE SINGULAR CARDINAL HYPOTHESIS
}

\author{
W. J. MITCHELL
}

\begin{abstract}
We use core model theory to obtain the following lower bounds to the consistency strength for the failure of the Singular Cardinal Hypothesis: Suppose that $\kappa$ is a singular strong limit cardinal such that $2^{\kappa}>\kappa^{+}$. Then there is an inner model $K$ such that $o(\kappa)=\kappa^{++}$in $K$ if $\kappa$ has uncountable cofinality, and $\forall \alpha<\kappa \exists \nu<\kappa o(\kappa) \geq \nu$ in $K$ otherwise.
\end{abstract}

\section{INTRODUCTION}

The Singular Cardinal Hypothesis $(\mathrm{SCH})$ asserts that if $\kappa$ is any singular strong limit cardinal then $2^{\kappa}=\kappa^{+}$. It is known to be consistent that the SCH fails: Prikry [Pr] obtains a model of $\neg \mathrm{SCH}$ from a model in which the GCH fails at a measurable cardinal $\kappa$, and Silver in turn (see [KM]) obtains the failure of the $\mathrm{GCH}$ at a measurable cardinal from a model for a $\kappa^{+}$-supercompact cardinal. Silver's result has been improved by Woodin [W] and again by Gitik [G] so that the consistency of " $\kappa$ measurable and $2^{\kappa}>\kappa^{+}$", and hence of $\neg \mathrm{SCH}$, is now known to follow from that of $o(\kappa)=\kappa^{++}$. Results in [Mi?] show that the failure of the $\mathrm{GCH}$ at a measurable cardinal is in fact equiconsistent with $\exists \kappa o(\kappa)=\kappa^{++}$.

The best previously published lower bounds on the consistency strength of $\neg \mathrm{SCH}$ are those of Dodd and Jensen, [D] which use the covering lemma for $L^{\mu}$ to show that at least one measurable cardinal (and slightly more) is required. In this paper we use the core model $K(\mathscr{F})$ for sequences of measures [Mi84b, Mi?, Mi87] to prove

1.1 Theorem. (i) $\operatorname{Con}(\neg S C H)$ implies $\operatorname{Con}(\exists \kappa \forall \alpha<\kappa \exists \nu<\kappa o(\nu) \geq \alpha)$.

(ii) If $\kappa$ is a singular strong limit cardinal with $\operatorname{cf}(\kappa)>\omega$ and $2^{\kappa}>\kappa^{+}$then there is an inner model with $o(\kappa)=\kappa^{++}$.

Since this paper was originally submitted Gitik has improved this result to give the exact consistency strength of the failure of the SCH. In [G?] he strengthens the conclusion of clause (i) to $o(\kappa)=\kappa^{++}$, which is shown in [G] to be best

Received by the editors March 27, 1990.

1980 Mathematics Subject Classification (1985 Revision). Primary 03E35, 03E45, $03 E 55$.

Key words and phrases. Core model, covering lemma, $\mathrm{GCH}, \mathrm{SCH}$.

Some of the work in this paper was done while the author was visiting the Hebrew University with support from the Lady Davis Foundation, and while the author was visiting UCLA and the California Institute of Technology.

This work was partially supported by grant number DMS-8614447 from the National Science Foundation. 
possible. The arguments of [G?] use results from $\S 2$ of this paper but his proof, which uses results of Shelah, is substantially different from our arguments in $\S 4$. Our proof gives some additional information in the limited situation in which it applies and may be of some independent interest. In [G??] he strengthens the conclusion of Theorem 1.1(ii) to $o(\kappa)=\kappa^{++}+\lambda$, where $\lambda=\operatorname{cf}(\kappa)$. This result is best possible, as is shown by a generalization by McDermitt [Mc] of Woodin's work. In this case Gitik does use our argument from $\S 3$ of this paper, with one new technique which overcomes the difficulty encountered in working with extenders in place of measures.

Our proof of Theorem 1.1 depends on a refined version of the covering lemma for sequences of measures from [Mi?, §6]. Theorem 1.2 below is the basic statement of this refinement, but we will need more detailed information about the system $\mathscr{C}$ of indiscernibles. This information appears in $\S 2$ along with the proof of Theorem 1.2. The covering set $h^{\prime \prime}(\gamma ; \mathscr{C})$ in the statement of the lemma is the smallest set containing $\gamma$, closed under $h$, and containing $\mathscr{C}(\alpha, \beta)$ whenever it contains $\alpha$ and $\beta$.

1.2 Theorem. Assume that there is no inner model of $\exists \kappa o(\kappa)=\kappa^{++}$. Then for any set $w$ of ordinals there is a function $h \in K(\mathscr{F})$, an ordinal $\gamma<\left|w^{\omega}\right|^{+}$, and a system $\mathscr{C}$ of indiscernibles for $K(\mathscr{F})$ such that $h^{\prime \prime}(\gamma ; \mathscr{C}) \supset w$.

This version is much closer to the covering lemmas for $L$ and $L^{\mu}$, proved by Jensen and by Jensen and Dodd respectively, than that given in [Mi?], but it is weaker than those versions in one crucial respect: a direct generalization of their results would give a single system $\mathscr{C}$ which works for all sets $w$. This generalization is known to be false, (see the discussion following the proof of Theorem 4.1 in $\S 4$ ) but the system $\mathscr{C}$ given by Theorem 1.2 is, in a sense to be made precise later, unique and maximal up to finite changes for the measures which lie in the covering set $h^{\prime \prime}(\gamma ; \mathscr{C})$. The proof of Theorem 1.1 will depend on using this maximality and uniqueness to define a small set of "standard" systems of indiscernibles which is large enough that Theorem 1.2 is still true if the system $\mathscr{C}$ is required to be taken from this set.

The Singular Cardinal Hypothesis can be stated in a more general form which does not assume that $\kappa$ is a strong limit cardinal: If $\kappa$ is any singular cardinal then $\kappa^{\mathrm{cf}(\kappa)}=\max \left(\kappa^{+}, 2^{\mathrm{cf}(\kappa)}\right)$. Dodd and Jensen's results in [D] are still valid for this stronger statement of the $\mathrm{SCH}$. The same is probably true of Theorem 1.1 but a proof would require using techniques of Dodd and Jensen to avoid the assumption, frequently used both in [Mi?] and in this paper, that the set $X \prec$ $H_{\kappa^{++}}$used in the proof of the covering lemma is closed under $\omega$ sequences.

The proof of Theorem 1.2, together with the analysis of the maximality of the sequence of indiscernibles, is in $\S 2$. $\S 3$ contains the proof of Theorem 1.1(ii), the case of uncountable cofinality, and $\S 4$ contains the proof of Theorem 1.1(i), the case of countable cofinality. Each of the $\S \S 3$ and 4 depends on $\S 2$, but they can be read independently of each other.

This paper depends heavily on the results and methods of [M84b and M?]. We have attempted to summarize the necessary facts in this paper, but an acquaintance with that material would be desirable. At the least an understanding of the theory of coherent sequences of measures [Mi74, Mi83] is necessary. We summarize below some of the notation from these sources. 
Notation. A sequence of measures is a coherent function $\mathscr{F}$ with domain of the form $\left\{(\alpha, \beta): \alpha<l^{\mathscr{F}}\right.$ and $\left.\beta<o^{\mathscr{F}}(\alpha)\right\}$, where $l^{\mathscr{F}}$ is an ordinal and $o^{\mathscr{F}}$ is a function, such that $\mathscr{F}(\alpha, \beta)$ is a measure on $\alpha$ for all ordinals $\alpha<l^{\mathscr{F}}$ and $\beta<o^{\mathscr{F}}(\alpha)$. The requirement that $\mathscr{F}$ be coherent means that for all ordinals $\alpha, \beta^{\prime}$ and $\beta$ such that $\alpha<l^{\mathscr{F}}$ and $\beta^{\prime}<\beta<o^{\mathscr{F}}(\alpha)$, if $f \in K(\mathscr{F})$ is the least function such that $[f]_{\mathscr{F}_{(\alpha, \beta)}}=\beta^{\prime}$ then for all $x \in \mathscr{P}(\alpha) \cap K(\mathscr{F})$ we have $x \in \mathscr{F}\left(\alpha, \beta^{\prime}\right)$ iff $\{\nu: x \cap \nu \in \mathscr{F}(\nu, f(\nu))\} \in \mathscr{F}(\alpha, \beta)$. We write $\mathfrak{C}\left(\alpha, \beta^{\prime}, \beta\right)$ for this coherence function $f$. The coherence of $\mathscr{F}$ implies that if $i: K(\mathscr{F}) \rightarrow$ $\operatorname{ult}(K(\mathscr{F}), \mathscr{F}(\alpha, \beta))$ is the canonical embedding then $i(\mathscr{F})\left(\alpha, \beta^{\prime}\right)=\mathscr{F}\left(\alpha, \beta^{\prime}\right)$ for all $\beta^{\prime}<\beta<o^{\mathscr{F}}(\alpha)$.

The precise definition of a system of indiscernibles is given in the next section as Definition 2.5, but we present here a review of the basic theory. A system of indiscernibles for $\mathscr{F}$ is a function $\mathscr{C}$ with domain $(\mathscr{C}) \subset \operatorname{domain}(\mathscr{F})$ such that $\mathscr{C}(\alpha, \beta)$ is a subset of $\alpha$ whenever $(\alpha, \beta) \in$ domain $(\mathscr{C})$. As one would expect, the sets $\mathscr{C}(\alpha, \beta)$ resemble Prikry sequences in the sense that if $\mathscr{C}(\alpha, \beta)$ is cofinal in $\alpha$ then

$$
\forall x \in \mathscr{P}(\alpha) \cap K(\mathscr{F})(x \in \mathscr{F}(\alpha, \beta) \Leftrightarrow(\mathscr{C}(\alpha, \beta) \backslash x \text { is bounded in } \alpha))
$$

but the ordinals in $\mathscr{C}$ also work uniformly as indiscernibles, even when they belong to different measures: if $\vec{c}, \vec{\alpha}$, and $\vec{\beta}$ are $\omega$-sequences such that $\vec{c}$ is increasing, $c_{i} \in \mathscr{C}\left(\alpha_{i}, \beta_{i}\right)$ for all $i \in \omega$, and $\bigcup_{i} c_{i}=\bigcup_{i} \alpha_{i}$, then for any function $g \in K(\mathscr{F})$ there is $i_{0}<\beta$ such that for all $i>i_{0}$

$$
\forall x \in g^{\prime \prime} c_{i}\left(c_{i} \in x \Leftrightarrow x \in \mathscr{F}\left(\alpha_{i}, \beta_{i}\right)\right) .
$$

The systems of indiscernibles used in this paper will come, directly or indirectly, from iterated ultrapowers. Since we are making one minor change from [Mi84b] in the construction of a system of indiscernibles from an iterated ultrapower, we give the full definition here. Suppose, in general, that $i_{0, \theta}: M_{0} \rightarrow M_{\theta}$ is an iterated ultrapower, with $M_{\alpha+1}=\operatorname{ult}\left(M_{\alpha}, \mathscr{F}_{\alpha}\left(\kappa_{\alpha}, \beta_{\alpha}\right)\right)$ for each $\alpha<\theta$ where $\mathscr{F}_{0}$ is a coherent sequence of measures in $M_{0}, \mathscr{F}_{\alpha}=i_{0, \alpha}\left(\mathscr{F}_{0}\right)$, and the sequence $\kappa_{\alpha}$ is strictly increasing. For $\kappa \in \operatorname{range}\left(i_{0, \alpha}\right)$, we define

$$
\mathscr{C}_{\alpha}(\kappa, \beta)=\left\{\kappa_{\alpha^{\prime}}: \kappa=i_{\alpha^{\prime}, \alpha}\left(\kappa_{\alpha^{\prime}}\right) \text { and } \beta=i_{\alpha^{\prime}, \alpha}\left(\beta_{\alpha^{\prime}}\right)\right\} \text {. }
$$

If $\kappa \notin$ range $i_{0, \alpha}$ then $\mathscr{C}_{\alpha}(\kappa, \beta)$ is defined to be empty unless $\kappa \in \mathscr{C}_{\alpha}(\lambda, \gamma)$ for some $\lambda \in \operatorname{range}\left(i_{0, \alpha}\right)$, in which case let $\beta^{\prime}=i_{\alpha^{\prime}, \alpha}(\beta)$ where $\kappa=\kappa_{\alpha^{\prime}}$, so that $\beta^{\prime}<\gamma$ and $\beta=\mathfrak{C}\left(\lambda, \beta^{\prime}, \gamma\right)(\kappa)$. Then we set

$$
\mathscr{C}_{\alpha}(\kappa, \beta)=\mathscr{C}_{\alpha}\left(\lambda, \beta^{\prime}\right) \cap\left(\kappa \backslash \kappa_{\xi}\right)
$$

where $\xi<\alpha$ is the least ordinal such that $\gamma \in \operatorname{range}\left(i_{\xi, \alpha}\right)$. The definition given in [Mi84b] was equivalent except that $\kappa_{\xi}$ was omitted, so that in the second case $\mathscr{C}_{\alpha}(\kappa, \beta)$ would be $\mathscr{C}_{\alpha}\left(\lambda, \beta^{\prime}\right) \cap \kappa$.

Suppose that $\mathscr{C}=\mathscr{C}_{\theta}$ is a system of indiscernibles constructed as above. Some basic consequences of this construction are that $\bigcup_{\beta} \mathscr{C}(\alpha, \beta)$ is closed in $\alpha$ and that if $\beta \neq \beta^{\prime}$ then $\mathscr{C}(\alpha, \beta) \cap \mathscr{C}\left(\alpha, \beta^{\prime}\right)=\varnothing$. If $c$ is an indiscernible in $\mathscr{C}$ then there will be a unique pair $(\alpha, \beta)$ such that $\alpha$ is not an indiscernible and $c \in \mathscr{C}(\alpha, \beta)$. We will write $\alpha^{\mathscr{C}}(c)$ and $\beta^{\mathscr{C}}(c)$ for this pair of ordinals. These ordinals have another equivalent characterization: if $c=\kappa_{\nu}$ in the iteration then $\alpha^{\mathscr{E}}(c)$ is the least member of $\operatorname{range}\left(i_{\nu}, \theta\right) \backslash c$ and $\beta^{\mathscr{E}}(c)$ is the unique 
member $\beta$ of range $\left(i_{\nu, \theta}\right) \cap o(\alpha)$ such that for all $x \in \operatorname{range}\left(i_{\nu, \theta}\right) \cap \mathscr{P}\left(\alpha^{\mathscr{C}}(c)\right)$ we have $c \in x \Leftrightarrow x \in \mathscr{F}_{\theta}\left(\alpha^{\mathscr{C}}(c), \beta\right)$.

Now suppose that $m_{0}$ is a mouse with projectum $\rho$ and $i=i_{0, \theta}: m_{0} \rightarrow$ $m=m_{\theta}$ is an iterated ultrapower such that $i\left\lceil\rho\right.$ is the identity. Let $h_{0}$ be the canonical skolem function for $m_{0}$. Then $i$ maps $h_{0}$ to the canonical skolem function $h$ for $m$, so that $h^{\prime \prime} \rho=\operatorname{range}(i)$ and in general if $c=\kappa_{\nu}$ then $h^{\prime \prime} c=\operatorname{range}\left(i_{\nu, \theta}\right)$. Hence the characterization of the functions $\alpha^{\mathscr{E}}$ and $\beta^{\mathscr{E}}$ may be given in a form that depends, apart from their domain, only on the function $h$; namely $\alpha^{\mathscr{E}}(c)=\min \left(h^{\prime \prime} c \backslash c\right)$ and $\beta^{\mathscr{C}}(c)$ is the unique ordinal $\beta \in h^{“} c \cap o\left(\alpha^{\mathscr{C}}(c)\right)$ such that

$$
\forall x \in h^{“} c \cap \mathscr{P}\left(\alpha^{\mathscr{C}}(c)\right)\left(c \in x \Leftrightarrow x \in \mathscr{F}\left(\alpha^{\mathscr{E}}(c), \beta\right)\right) .
$$

Following this idea, we use this last characterization of $\alpha^{\mathscr{C}}$ and $\beta^{\mathscr{C}}$ to define functions $\alpha^{m}(\nu)$ and $\beta^{m}(\nu)$ for all ordinals $\nu$ in $m$ rather than only for indiscernibles $c$ in $\mathscr{C}$. Thus the functions $\alpha^{m}$ and $\beta^{m}$ are definable over the mouse $m$, but the functions $\alpha^{\mathscr{C}}$ and $\beta^{\mathscr{C}}$ are equal to $\alpha^{m}\left\lceil C\right.$ and $\beta^{m} \mid C$, respectively, where $C=\bigcup_{\alpha, \beta} \mathscr{E}(\alpha, \beta)$. In the special case when $\alpha^{m}(\nu)=\nu$ or there is no ordinal $\beta$ satisfying formula (1) we set $\beta^{m}(\nu)=o^{m}(\nu)$. In particular $\alpha^{m}(\nu)=\nu$ and $\beta^{m}(\nu)=o(\nu)$ whenever $\nu \in h^{\prime \prime} \nu$.

The system of indiscernibles $\mathscr{C}$ given by the covering Lemma 1.2 will be constructed indirectly from an iterated ultrapower, and as a result the idea of the last paragraph will apply, except that $m$ will be a $\mathscr{F} \mid \kappa$-mouse and hence a member of $K(\mathscr{F})$. The construction of a system of indiscernibles from an iterated ultrapower was changed from that of [Mi84b] so that $\mathscr{C}$ would be a system of indiscernibles for $K(\mathscr{F})$ as well as for $m$. To see why the change is necessary, note that since $m \in K(\mathscr{F})$ the functions $\alpha^{m}$ and $\beta^{m}$ are members of $K(\mathscr{F})$. Now define a function $k$ on the ordinals of $m$ by letting $k(\nu)$ be the least ordinal $\delta<\nu$ such that $\beta^{m}(\nu) \in h^{\prime \prime} \delta$. Then $k \in K(\mathscr{F})$, and hence indiscernibility requires that (with at most finitely many exceptions) we have $k(\alpha)<c<\alpha$ whenever $c \in \mathscr{C}(\alpha, \beta)$. The change in the construction insures that this requirement is satisfied.

If $x$ is any member of $m$ then $x$ has a minimal support $d$ in the iterated ultrapower, that is, there is a finite set $d$ of indiscernibles from the system $\mathscr{C}$ generated by $i$ such that $x \in h^{\prime \prime}(\rho \cup d)$, and such that $d$ is contained in any other set $d^{\prime}$ of indiscernibles such that $x \in h^{\prime \prime}\left(\rho \cup d^{\prime}\right)$.

The expression $K(\mathscr{F})$ will always denote the maximal core model for sequences of measures as defined in [Mi?]. A $\mathscr{F} \mid \kappa$-mouse is a model $m=$ $J_{\xi}\left(\mathscr{F}^{m}\right)$, with $\mathscr{F}^{m}|\kappa=\mathscr{F}| \kappa$ and $o^{\mathscr{F}}(\alpha)<\kappa$ for $\alpha<\kappa$, such that $m$ is iterable and every member of $m$ is definable in $m$ from parameters in $\kappa \cup p^{m}$ for some finite set $p^{m}$ of ordinals. The ordinal $\kappa$ is refered to as the projectum of $m$. It should be noted that the coherence function $\mathfrak{C}^{m}$ for $\mathscr{F} m$ will be different from the coherence function $\mathfrak{C}$ for $\mathscr{F}$, but since $\mathscr{F}^{m}\lceil\kappa=\mathscr{F}\lceil\kappa$ we have $\mathfrak{C}^{m}\left(\alpha, \beta^{\prime}, \beta\right)=\mathfrak{C}\left(\alpha, \beta^{\prime}, \beta\right)$ whenever $\alpha<\kappa$. We will not be using $\mathfrak{C}^{m}\left(\alpha, \beta^{\prime}, \beta\right)$ for ordinals $\alpha>\kappa$, so it follows that the problem only arises for $\alpha=\kappa$.

For the benefit of those with some acquaintance with the fine structure sections of [Mi84b] it should be acknowledged that some of our discussion has been somewhat sloppy. In the notation from that paper, a mouse $m_{0}$ is actually the 
$\Sigma_{n}^{*}$-code of a structure of the form $J_{\xi_{0}}\left(\mathscr{F}^{m_{0}}\right)$, for some $n \in \omega$, and an iterated ultrapower of $m_{0}$ is the internal ultrapower of the $\Sigma_{n}^{*}$-code $m_{0}$. This iterated ultrapower may be regarded as a $\Sigma_{n}^{*}$-ultrapower of $J_{\xi_{0}}\left(\mathscr{F} m_{0}\right)$ which has all of its critical points $\kappa_{\alpha}$ in the interval between the $(n+1)$ th and $n$th projectums of $J_{\xi_{\alpha}}\left(\mathscr{F}^{m_{\alpha}}\right)$.

We write $H_{\kappa}$ for the sets hereditarily of cardinality less than $\kappa$, and if $M$ is a model of set theory then we write $H_{\kappa}^{M}$ for $H_{\kappa}$ as defined in $M$.

\section{THE COVERING LEMMA}

In this section we will prove Theorem 1.2, together with various results giving more information about the system $\mathscr{C}$ of indiscernibles. For background, and in order to provide a framework and notation for our work, we begin with an outline of the proof of the covering lemma from [Mi?]. Many of the ideas of this section were previously used in [Mi87]. The results from this section will be used in the rest of the paper to show that there is a collection of at most $\kappa^{+}$ systems of indiscernibles which is rich enough to cover all small subsets of $\kappa$. The Singular Cardinal Hypothesis will then follow from the fact that most $\kappa^{+}$ sets can be covered using only $\kappa^{+}$systems.

Let $w$ be the set to be covered, let $\kappa=\sup (w)$, and assume wlog that $|w|^{\omega}<|\kappa|$. We can also assume wlog that $\kappa$ is a cardinal in $K(\mathscr{F})$. Instead of working with $w$ directly, pick a set $X \supset w$ such that $X \prec H_{\kappa^{++}},{ }^{\omega} X \subset X$, and $|X|=|w|^{\omega}$. We will obtain a covering of $X$ which satisfies all of the conditions of Theorem 1.2 except that the condition $\gamma<\left|w^{\omega}\right|^{+}$is replaced by the weaker condition $\gamma<\kappa$. Theorem 1.2 follows from this weaker result by a simple induction.

The first part of this section outlines the construction from [Mi?] of the $\mathscr{F}\left\lceil\kappa\right.$-mouse $m^{X}$ and system $\mathscr{C}^{X}$ of indiscernibles for $m$ such that $X$, and hence $w$, can be covered using $m$ and $\mathscr{C}^{X}$. The rest of the section will contain results showing that $\mathscr{C}^{X}$ is a system of indiscernibles for $K(\mathscr{F})$ rather than just for the mouse $m$, and finally that the system $\mathscr{C}^{X}$ is, in an appropriate sense, unique and maximal.

The basic proof of the covering lemma, as given in [Mi?], is as follows: Let $N$ be the transitive collapse of $X$ and let $\pi: N \cong X \prec H_{\kappa^{++}}$be the isomorphism. We will use an overbar to indicate a preimage under the map $\pi$, so that for example $\pi(\bar{\kappa})=\kappa$ and $\pi(\bar{F})=\mathscr{F} \mid \kappa$. Since $|X|<|\kappa|$ and $X$ is cofinal in $\kappa, \pi$ is not the identity on $\bar{\kappa}$. Now let $\delta$ be the critical point of $\pi$. If $\mathscr{P}(\delta) \cap K(\mathscr{F}) \subset N$ then $U=\{x \in \mathscr{P}(\delta) \cap K(\mathscr{F}): \delta \in \pi(x)\}$ is an ultrafilter which is not in $K(\mathscr{F})$. Now $U$ is countably complete since ${ }^{\omega} N \subset N$, so $U$ could have been included in the sequence $\mathscr{F}$. The only reason why it would not have been included is if $K(\mathscr{F})$ already satisfied that $o^{\mathscr{F}}(\delta)=\delta^{++}$, which would contradict the assumption that there is no model with such a cardinal $\delta$. It follows that $\mathscr{P}(\delta) \cap K(\mathscr{F}) \not \subset N$ and since $\mathscr{F}|\delta=\overline{\mathscr{F}}| \delta$ it follows that there is an $\overline{\mathscr{F}} \mid \delta$-mouse which is not in $N$. Let $\bar{m}_{0}$ be the least mouse not in $N$, that is, $\bar{m}_{0}$ is a $\bar{F} \mid \xi$-mouse for some $\xi<\bar{\kappa}$ and there is no smaller $n$ which is a $\bar{F} \mid \xi^{\prime}$-mouse for any $\xi^{\prime}<\kappa$. Then there is an iterated ultrapower $i_{0, \theta}: \bar{m}_{0} \rightarrow \bar{m}_{\theta}=\bar{m}$ so that $\mathscr{F}^{\bar{m}} \mid \bar{\kappa}=\overline{\mathscr{F}}$. The minimality of $\bar{m}_{0}$ implies that $\left(H_{\bar{\kappa}}\right)^{\bar{m}} \subset N$, and the embedding $i_{0, \theta}$ generates a system $\overline{\mathscr{C}}$ of indiscernibles for $\bar{m}$. 
Because $\left(H_{\bar{\kappa}}\right)^{\bar{m}} \subset N$ we can extend $\pi\left\lceil\bar{\kappa}\right.$ to a map $\pi^{*}: \bar{m} \rightarrow m$ where $m=$ $m^{X}$ is an $\mathscr{F}\left\lceil\kappa\right.$-mouse. Again using ${ }^{\omega} N \subset N, m$ is iterable and hence is a member of $K(\mathscr{F})$. Then the system $\mathscr{C}^{X}$ defined by setting $\mathscr{C}^{X}\left(\pi^{*}(\alpha), \pi^{*}(\beta)\right)$ $=\pi^{“}(\overline{\mathscr{C}}(\alpha, \beta))$ is a system of indiscernibles for $m^{X}$, and the canonical skolem function $\bar{h}$ for $\bar{m}$ maps to the canonical skolem function $h^{m}$ for $m^{X}$. Let $\rho=\pi(\bar{\rho})$. Then $\rho<\kappa$. Also $\bar{m}=\bar{h}^{\prime \prime}(\bar{\rho} ; \overline{\mathscr{C}})$ since $\bar{\rho}$ is the projectum of $\bar{m}$, and it follows that $w \subset X \cap \kappa=\pi^{* \prime}(\bar{m} \cap \bar{\kappa})=h^{m "}\left(\pi^{\prime \prime}(\bar{\rho}) ; \mathscr{C}^{X}\right) \cap \kappa \subset h^{m^{\prime \prime}}\left(\rho ; \mathscr{C}^{X}\right)$.

If $d \subset \kappa$ then we will generally abuse notation by writing $h^{m \text { "s }}(d)$ for $h^{\prime \prime}[\rho \cup d]^{<\omega}$.

The projectum of $m^{X}$ is equal to $\kappa$ rather than $\rho$, and in fact $H_{\kappa} \cap K(\mathscr{F}) \subset$ $m$. To see this note that $\mathscr{P}(\bar{\kappa}) \cap N \cap K(\overline{\mathscr{F}}) \subset \bar{m}$ since $\bar{m}$ is larger than any mouse which is a member of $N$. But $\pi^{*}$ is equal to $\pi$ on $\mathscr{P}(\bar{\kappa}) \cap N$, so $\mathscr{P}(\kappa) \cap K(\mathscr{F}) \cap X \subset m$. The elementarity of $X$ implies that it contains a subset $x$ of $\kappa$ which is is constructed later in the canonical order of construction of $K(\mathscr{F})$ than any bounded subset of $\kappa$. It follows that $x \in m$ and hence every bounded subset of $\kappa$ in $K(\mathscr{F})$ is in $m$.

One complication should be mentioned here. The system $\mathscr{C}^{X}$ is a system of indiscernibles for the sequence $\mathscr{F}^{m}$ of measures in $m$, rather than for the sequence $\mathscr{F}$ of $K(\mathscr{F})$. This means that the domain of $\mathscr{C}^{X}$ is equal to the domain of $\mathscr{F} \mathrm{m}$, rather than to the domain of $\mathscr{F}$. This does not matter below $\kappa$, since $\mathscr{F}^{m}\left\lceil\kappa=\mathscr{F}\left\lceil\kappa\right.\right.$, but if $o(\kappa) \geq \kappa^{+}$then $\mathscr{F}^{m}$ and $\mathscr{F}$ will differ at $\kappa$. Let $A$ be defined in $K(\mathscr{F})$ as $\left\{\nu: o(\nu)=\nu^{+}\right\}$. Then the unique $\eta$ such that $A \in \mathscr{F}^{m}(\kappa, \eta)$ will be $\kappa^{+m}$ and the unique $\eta$ such that $A \in \mathscr{F}(\kappa, \eta)$ will be $\kappa^{+}$. Thus $\mathscr{F}^{m}\left(\kappa, \kappa^{+m}\right)$ extends naturally to $\mathscr{F}\left(\kappa, \kappa^{+}\right)$rather than to $\mathscr{F}\left(\kappa, \kappa^{+m}\right)$, even though $|m|=\kappa$ implies that $\kappa^{+m}<\kappa^{+}$.

If $o(\kappa)<\kappa^{+}$then this consideration is not a problem, since in that case $\mathscr{F}$ and $\mathscr{F}^{m}$ are equal at $\kappa$. This may be proved by using iterated ultrapowers to compare the models $m$ and $\operatorname{ult}(m, \mathscr{F}(\kappa, \beta))$ for $\beta<o^{\mathscr{F}}(\kappa)$, using the fact that for $\beta<\kappa^{+(m)}$ the coherence functions $\mathfrak{C}\left(\kappa, \beta^{\prime}, \beta\right)$ of $K(\mathscr{F})$ are in $m$. In particular, this applies to the proof of Theorem 1.1(i) in $\S 4$, where there is a fixed bound $\beta_{0}$, smaller than the first relevant measurable cardinal, for the order $o(\alpha)$ of any measurable cardinal $\alpha$. This bound also makes unnecessary many of the complications of this section. It means that if $c \in \mathscr{C}(\alpha, \beta)$ then $\beta=o(c)$, and since it can be assumed that $\beta_{0}$ is contained in $X$ there is no concern about the definability of $\beta<\beta_{0}$.

Most of the rest of this section will be concerned with looking in more detail at the structure of the covering set $h^{m^{\prime \prime}}\left(\delta ; \mathscr{C}^{X}\right)$. We will generally write $m$ for $m^{X}$, and in general will frequently drop superscripts when they are not necessary to prevent ambiguity.

2.1 Definition. A finite increasing sequence $d=\left(d_{0}, \ldots, d_{n-1}\right)$ of ordinals is a weak support in $\mathscr{C}^{X}$ if for each $i<n$ there are $\alpha_{i}, \beta_{i} \in h^{\prime \prime}(d\lceil i)$ such that $d_{i} \in \mathscr{C}^{X}\left(\alpha_{i}, \beta_{i}\right)$.

2.2 Lemma. For every set $y \in \operatorname{range}\left(\pi^{*}\right)$ there is a weak support $d$ such that $y \in h^{\prime \prime}\left(\rho^{X} \cup d\right)$.

Proof. Since every such set $y$ has the form $h(\nu)$ for some ordinal $\nu$, it is sufficient to prove the lemma for the case where $y=\nu$ is an ordinal. The 
proof is by induction on $\nu$. If $\nu$ is not an indiscernible then $\nu=h\left(\nu^{\prime}\right)$ for some $\nu^{\prime}<\nu$, and the induction hypothesis implies that there is a sequence $d \subset \nu^{\prime}+1 \subset \nu$ which is a weak support for $\nu^{\prime}$ and hence for $\nu$. If $\nu$ is an indiscernible then $\alpha^{m}(\nu)$ and $\beta^{m}(\nu)$ are in $h^{\text {"“ }} \nu$ and it follows by the induction hypothesis that they have a weak support $d \subset \nu$. Then $d \cup\{\nu\}$ is a support for $\nu$.

For Lemma 2.4 we need one more fact using the fine structure:

2.3 Lemma. The skolem function $h$ for $m$ is the increasing union of a set of partial functions $h^{\xi}$ such that each $h^{\xi}$ is a member of $m$. Cofinally many of the functions $h^{\xi}$ are of the form $\pi^{*}\left(\bar{h}_{\bar{\xi}}\right)$ where $\bar{h}^{\bar{\xi}}$ is contained in $\bar{h}$, and cofinally many of the functions $h^{\xi}$ are in $h^{m}$ " $\rho^{X}$.

Proof. The existence of the functions $h^{\xi}$ depends on the fine structure of $m$. We will give the definition for the case in which $m$ is a $\Sigma_{1}$-code, that is, $m=$ $J_{\alpha}\left(\mathscr{F}^{m}\right)$ for some ordinal $\alpha$ and $h$ is the $\Sigma_{1}$ skolem function for $J_{\alpha}(\mathscr{F} m)$ with parameter $p=p_{1}^{m}$. The construction is similar for the general case of a $\Sigma_{n}^{*}$ code but does, of course, require knowledge of the $\Sigma_{n}^{*}$-codes of a structure $J_{\alpha}\left(\mathscr{F}^{m}\right)$.

In the $\Sigma_{1}$ case the skolem function $h$ for $m$ is the partial function defined in $J_{\alpha}\left(\mathscr{F}^{m}\right)$ by

$$
h(\nu)=x \Leftrightarrow \exists y\left(R(\nu, x, p, y) \wedge \forall\left(x^{\prime}, y^{\prime}\right)<^{m}(x, y) \neg R\left(\nu, x^{\prime}, p, y^{\prime}\right)\right)
$$

where $\exists y R$ is the universal $\Sigma_{1}$ formula and $<^{m}$ is the order of construction of $J_{\alpha}(\mathscr{F} \mathrm{m})$. If $\alpha$ is a limit ordinal and $\xi<\alpha$ then we define $h^{\xi}$ for $\xi<\alpha$ by $h^{\xi}(\nu)=x$ if and only if

$$
\begin{aligned}
x \in J_{\xi}\left(\mathscr{F}^{m}\right) \wedge \exists y \in & J_{\xi}\left(\mathscr{F}^{m}\right) \\
& \left(R(\nu, x, p, y) \wedge \forall\left(x^{\prime}, y^{\prime}\right)<^{m}(x, y) \neg R\left(\nu, x^{\prime}, p, y^{\prime}\right)\right) .
\end{aligned}
$$

If $\alpha=\gamma+1$ is a successor ordinal then $h^{\xi}(\nu)$ is defined for integers $\xi<\omega$ by setting $h^{\xi}(\nu)=x$ if and only if

$$
\begin{aligned}
x \in S_{\gamma^{*} \omega+\xi}\left(\mathscr{F}^{m}\right) \wedge & \exists y \in S_{\gamma^{\cdot} \omega+\xi}(\overline{\mathscr{F}}) \\
& \left(R(\nu, x, p, y) \wedge \forall\left(x^{\prime}, y^{\prime}\right)<^{m}(x, y) \neg R\left(\nu, x^{\prime}, p, y^{\prime}\right)\right),
\end{aligned}
$$

where $S_{\gamma^{\circ} \omega+\xi}$ is the union of the images of $J_{\gamma}(\mathscr{F}) \cup\left\{J_{\gamma}\left(\mathscr{F}^{m}\right)\right\}$ under the first $\xi$ of the $\mathscr{F}^{m}$-rudimentary functions. In either case it is clear that the functions $h^{\xi}$ form an increasing sequence of partial functions, that each of the functions $h^{\xi}$ is in $m$, and that the union of this sequence is the skolem function $h$.

Let $\bar{h}^{\bar{\xi}}$ be defined in $\bar{m}$ in the same way that $h^{\xi}$ was defined in $m$. Since the skolem function $h$ for $m$ is the image under $\pi^{*}$ of the skolem function $\bar{h}$ for $\bar{m}$, we have $h^{\xi}=\pi^{*}\left(\bar{h}^{\bar{\xi}}\right)$, as required in the first half of the final sentence, whenever $\xi=\pi^{*}(\bar{\xi})$. If $\alpha$ is a limit ordinal then $\operatorname{range}\left(\pi^{*}\right)$ is cofinal in $\alpha$ since $\pi^{*}$ is defined as an extender using functions which are members of $\bar{m}$. If $\alpha$ is a successor ordinal then trivially every function $h^{\xi}$ for $\xi<\omega$ is in the range of $\pi^{*}$. This proves the first half of the last sentence of the lemma.

The second half of the final sentence uses a similar argument applied to the function $i_{0, \theta}$ instead of to $\pi^{*}$. If $\alpha$ is a limit ordinal then the range of $i_{0, \theta}$ is cofinal in $\bar{\alpha}$ since $i_{0, \theta}$ is an iterated ultrapower using functions in $\bar{m}_{0}$, and 
hence range $\left(\pi^{*} \cdot i_{0, \theta}\right)$ is cofinal in $\alpha$. Hence cofinally many of the functions $h^{\xi}$ are in the range of $\pi^{*} \cdot i_{0, \theta}$, and all such functions $h^{\xi}$ are in $h^{\text {" }} \rho^{X}$. Again, if $\alpha$ is a successor than all of the functions $h^{\xi}$ for $\xi<\omega$ are in the range of $\pi^{*} \cdot i_{0, \theta}$ and hence are in $h^{\prime \prime} \rho^{X}$.

2.4 Lemma. If $d$ is a weak support in $\mathscr{C}^{X}, c \in \mathscr{C}^{X}(\alpha, \beta)$, and $d \cap[c, \alpha)=\varnothing$ then for all $x \in h^{\prime \prime}(c \cup d \cup\{\alpha, \beta\})$ we have $c \in x$ iff $x \in \mathscr{F}^{m}(\alpha, \beta)$.

Proof. Suppose that the lemma is false, so that there is a set

$$
x \in h^{\prime \prime}(c \cup d \cup\{\alpha, \beta\})
$$

such that $c \in x \Leftrightarrow x \notin \mathscr{F}^{m}(\alpha, \beta)$. We begin by using the last lemma to show that the lemma is also false in $\bar{m}$ and we will then be able to reach a contradiction by using the fact that the system $\overline{\mathscr{C}}$ of indiscernibles for $\bar{m}$ is constructed from an iterated ultrapower.

Let $h^{\xi}$ be a function as in Lemma 2.3 such that $h^{\xi} \in \operatorname{range}\left(\pi^{*}\right)$ and $x \in$ $h^{\xi \text { “ }}(c \cup d \cup\{\alpha, \beta\})$. Then $m$ satisfies the sentence

$$
\exists x \in h^{\xi “(}(c \cup d \cup\{\alpha, \beta\})\left(c \in x \Leftrightarrow x \notin \mathscr{F}^{m}(\alpha, \beta)\right) .
$$

By elementarity, $\bar{m}$ satisfies the sentence

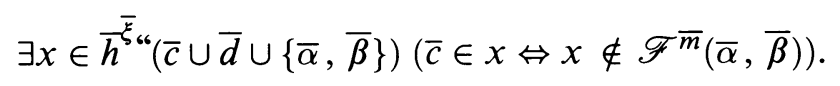

This gives the required counterexample in $\bar{m}$. Dropping the bars, this means that we have $c, \alpha, \beta, d$ and $x$ in $\bar{m}$ such that

$$
c \in \overline{\mathscr{C}}(\alpha, \beta), \quad d \cap[c, \alpha)=\varnothing, \quad x \in \bar{h}^{“}(c \cup d \cup\{\alpha, \beta\})
$$

and

$$
c \in x \Leftrightarrow x \notin \overline{\mathscr{F}}(\alpha, \beta) .
$$

Now we claim that $x \in \bar{h}^{\prime \prime}(c \cup d \cup\{\alpha\})$, so that $\beta$ can be omitted. If $\alpha=\alpha^{\bar{m}}(c)$ then this is immediate since $\beta=\beta^{\bar{m}}(c) \in \bar{h}^{\prime \prime} c$. If $\alpha \neq \alpha^{\bar{m}}(c)$ then $\alpha^{\bar{m}}(\alpha)=\alpha^{\bar{m}}(c)$, and by the construction of the system $\overline{\mathscr{C}}$ of indiscernibles for $\bar{m}$ we have $\beta^{\bar{m}}(\alpha) \in \bar{h}^{\prime \prime} c$ and $\beta=\mathbb{C}^{\bar{m}}\left(\alpha^{\bar{m}}(c), \beta^{\prime}, \beta^{\bar{m}}(\alpha)\right)(\alpha)$ for $\beta^{\prime}=$ $i_{\xi, \theta}(\beta)<\beta^{\bar{m}}(\alpha)$ in $\bar{h}^{\prime c} c$. Since $\alpha^{\bar{m}}(c), \beta^{\prime}$, and $\beta^{\bar{m}}(c)$ are all in $\bar{h}^{\prime \prime} c$ we have $\beta \in \bar{h}^{\prime \prime}(c \cup\{\alpha\})$ and hence $x \in \bar{h}^{\prime \prime}(c \cup d \cup\{\alpha\})$.

Now we claim that $d$ can also be omitted, so that $x \in \bar{h}^{\prime \prime}(c \cup\{\alpha\})$. Every member $y$ of an iterated ultrapower has a support which is minimal in the sense that it is contained in any support for $y$. Now if $\alpha=\kappa_{\nu}=\operatorname{crit}\left(i_{\nu, \theta}\right)$ then $x \subset \alpha$ implies that $x \in \operatorname{range}\left(i_{\nu+1, \theta}\right)$ and hence $x$ has a support which is contained in $\alpha+1$. On the other hand $x \in \bar{h}^{\prime \prime}(c \cup d \cup\{\alpha\})$ implies that there is a support contained in $c \cup d \cup\{\alpha\}$, and hence the minimal support must be contained in the intersection of the sets $\alpha+1$ and $c \cup d \cup\{\alpha\}$. Since $d \cap[c, \alpha)=\varnothing$ this intersection is equal to $c \cup\{\alpha\}$.

Now if $\alpha=\alpha^{\bar{m}}(c)$ then it follows immediately that $c \in x \Leftrightarrow x \in \mathscr{F}^{\bar{m}}(\alpha, \beta)$. If $\alpha<\alpha^{\bar{m}}(c)$ then $x \in \mathscr{F}^{\bar{m}}(\alpha, \beta)$ iff $i_{\nu, \theta}(x) \in \mathscr{F}^{\bar{m}}\left(\alpha^{\bar{m}}(c), \beta^{\bar{m}}(c)\right)$ iff $c \in$ $i_{\nu, \theta}(x)$ iff $c \in X$ since $i_{\nu, \theta}(\alpha)=\alpha^{\bar{m}}(c)$ and $i_{\nu, \theta}(\beta)=\beta^{\bar{m}}(c)$.

Our next goal is to show that $\mathscr{C}^{X}$ is a system of indiscernibles for $K(\mathscr{F})$ rather than just for $m^{X}$. We begin with $\omega$ sequences, and then use this special case to understand longer sequences. 


\subsection{Definition.}

(i) An increasing $\omega$-sequence $\vec{c}$ of ordinals is an indiscernible sequence (over $K(\mathscr{F})$ ) for $(\vec{\alpha}, \vec{\beta})$ iff for each function $g \in K(\mathscr{F})$ there is $i_{0} \in \omega$ such that

$$
\forall i>i_{0} \forall x \in g^{\prime \prime}\left(c_{i} \cup\left\{\alpha_{i}, \beta_{i}\right\}\right) \quad\left(x \in \mathscr{F}\left(\alpha_{i}, \beta_{i}\right) \Leftrightarrow c_{i} \in x\right)
$$

(ii) $\mathscr{D}$ is a system of indiscernibles for $K(\mathscr{F})$ if $\mathscr{D}$ is a function such that

(1) (a) $\operatorname{domain}(\mathscr{D}) \subset \operatorname{domain}(\mathscr{F})$, and

$$
\{\mathscr{C}(\alpha, \beta):(\alpha, \beta) \in \operatorname{domain}(\mathscr{D})\}
$$

is a family of disjoint subsets of $\alpha$ for each ordinal $\alpha$.

(2) (b) For all $(\alpha, \beta)$ in domain $(\mathscr{D})$, and for all $\nu \in \mathscr{D}(\alpha, \beta)$ and all $\gamma<o(\nu)$ there is $\gamma^{\prime}<\beta$ and $\xi<\nu$ such that $\mathscr{D}(\nu, \gamma) \backslash \xi=$ $\mathscr{D}\left(\alpha, \gamma^{\prime}\right) \cap[\xi, \nu)$.

(3) (c) If $c<\alpha$ is a limit point of $\bigcup_{\beta} \mathscr{D}(\alpha, \beta)$ of cofinality $\omega$ then $c \in \mathscr{D}(\alpha, \gamma)$ for some $\gamma$ such that $\bigcup_{\beta \geq \gamma} \mathscr{D}(\alpha, \beta)$ is bounded in $c$.

(4) (d) If $\vec{c}, \vec{\alpha}$ and $\vec{\beta}$ are sequences such that $\vec{c}$ is increasing, $c_{i} \in$ $\mathscr{D}\left(\alpha_{i}, \beta_{i}\right)$ for all $i \in \omega$ and $\bigcup_{i} c_{i}=\bigcup_{i} \alpha_{i}$ then $\vec{c}$ is an indiscernible sequence for $(\vec{\alpha}, \vec{\beta})$.

2.6 Lemma. (i) $\mathscr{C}^{X}\lceil\kappa$ is a system of indiscernibles for $K(\mathscr{F})$. Furthermore clauses (a) and (b) of the definition of a system of indiscernibles for $K(\mathscr{F})$ hold for all of $\mathscr{C}^{X}$, as do clauses (c) and (d) if $o(\kappa)<\kappa^{+}$.

(ii) Conversely, suppose that $\vec{c}$ is an indiscernible sequence for $(\vec{\alpha}, \vec{\beta})$, that $\vec{c}$ and $\vec{\alpha}$ are in $X$ and that either $\alpha_{i}<\kappa$ for all $i$ or $o(\kappa)<\kappa^{+}$. Then $c_{i} \in \mathscr{C}^{X}\left(\alpha_{i}, \beta_{i}\right)$ for all but finitely many $i \in \omega$.

Proof. We first prove that $\mathscr{C}^{X} \mid \kappa$ is a system of indiscernibles for $K(\mathscr{F})$. Clause (ii)(a) and (ii)(b) of Definition 2.5 are immediate for $\mathscr{C}^{X}$. In order to establish clause (ii)(c), let $\vec{c}$ be a strictly increasing sequence with $c_{i} \in \mathscr{C}^{X}\left(\alpha, \beta_{i}\right)$, and let $c=\bigcup_{i} c_{i}$. Note that since ${ }^{\omega} X \subset X$ we have $\vec{c} \in X$ and hence $c \in X$. We can assume that $\alpha$ is not an indiscernible, since otherwise if the assertion fails for $\alpha$ then it still fails if we replace $\alpha$ with $\alpha^{m}(\alpha)$, using the fact that $\beta^{m}(\alpha) \in h^{\prime \prime} c_{i} \subset h^{\prime \prime} c$. It follows from the construction of $\overline{\mathscr{C}}$ and the definition of $\mathscr{C}^{X}$ that $c \in \mathscr{C}^{X}(\alpha, \beta)$ for some $\beta<o(\alpha)$. Suppose wlog that $\beta \leq \beta_{i}$ for all $i<\omega$.

Let $\bar{c}, \bar{c}_{i}, \bar{\beta}$, and $\bar{\beta}_{i}$ be the preimages under $\pi^{*}$ of $c, c_{i}, \beta$ and $\beta_{i}$ respectively. Then there are $\nu_{i}<\theta$ such that $\bar{c}_{i}$ is the critical point of $i_{\nu_{i}, \theta}: \bar{m}_{\nu_{i}} \rightarrow \bar{m}=\bar{m}_{\theta}$, and $\bar{c}$ is the critical point of $i_{\nu, \theta}$ where $\nu=\sup _{i} \nu_{i}$. Let $\delta=o^{\bar{F}}(\bar{c})$, so that $\bar{m}_{\nu+1}=\operatorname{ult}\left(\bar{m}_{\nu}, \overline{\mathscr{F}}_{\nu}(\bar{c}, \delta)\right)$ and $\bar{\beta}=i_{\nu, \theta}(\delta)$. Then $\overline{\mathscr{F}}_{\nu}(\bar{c}, \delta) \notin N$, since otherwise it would be in $\bar{K}$ and hence would not have been used in the iteration. We will reach a contradiction by proving that $\overline{\mathscr{F}}_{\nu}(\bar{c}, \delta)$ is in $N$.

Suppose that $x \in \bar{m} \cap \mathscr{P}(\bar{c})$. Then $x=i_{\nu_{i}, \nu}\left(x \cap \bar{c}_{i}\right)$ for all sufficiently large $i<\omega$, and for these $i$ we have $x \in \mathscr{F}_{\nu}(\bar{c}, \delta)$ iff $x \cap \bar{c}_{i} \in \mathscr{F}_{\nu_{i}}\left(\bar{c}_{i}, \delta_{i}\right)$ where $i_{\nu_{i}, \nu}\left(\delta_{i}\right)=\delta$. If $\beta_{i}=\beta$ then it follows that $\bar{c}_{i} \in x$ if and only if $x \cap \bar{c}_{i} \in \mathscr{F}_{\nu_{i}}\left(c_{i}, \delta_{i}\right)$ if and only if $x \in \mathscr{F}^{\bar{m}}(\bar{c}, \delta)$. Thus if $\beta_{i}=\beta$ for infinitely 
many $i$ then $\mathscr{F}_{m}(\bar{c}, \delta)=\left\{x: \exists i_{0} \forall i>i_{0}\left(\bar{\beta}_{i}=\bar{\beta} \Rightarrow c_{i} \in x\right) \in N\right.$, so we can assume wlog that $\beta<\beta_{i}$ for all $i$. Then

$$
i_{\nu_{i}, \theta}\left(\delta_{i}\right)=i_{\nu, \theta}(\delta)=\bar{\beta}<\bar{\beta}_{i}=i_{\nu_{i}, \theta}\left(o^{\bar{m}}\left(\bar{c}_{i}\right)\right) \text {, }
$$

so $\overline{\mathscr{F}}_{\nu_{i}}\left(\bar{c}_{i}, \delta_{i}\right)=\overline{\mathscr{F}}\left(\bar{c}_{i}, \delta_{i}\right) \in N$. Then $\left(\overline{\mathscr{F}}\left(\bar{c}_{i}, \delta_{i}\right): i<\omega\right) \in N$ since ${ }^{\omega} N \subset N$, and hence $\left.\mathscr{F}_{\nu}(\bar{c}, \delta)=\left\{x \subset \bar{c}: \exists i_{0} \forall i>i_{0} x \cap \bar{c}_{i} \in \overline{\mathscr{F}}\left(\bar{c}_{i}, \delta_{i}\right)\right\}\right\} \in N$. This contradiction completes the proof of clause (ii)(c) of Definition 2.5.

We now establish condition (ii)(d) of Definition 2.5. Suppose that $c_{i} \in$ $\mathscr{C}^{X}\left(\alpha_{i}, \beta_{i}\right)$ for almost all $i$ and $\bigcup_{i} c_{i}=\bigcup_{i} \alpha_{i}$ but $\vec{c}$ is not an indiscernible sequence for $(\vec{\alpha}, \vec{\beta})$. The sequences $\vec{c}$ and $(\vec{\alpha}, \vec{\beta})$ are in $X$ since ${ }^{\omega} X \subset X$, and by elementarity the statement that $\vec{c}$ is not an indiscernible sequence for $(\vec{\alpha}, \vec{\beta})$ is true in $X$. Let $g \in X$ be a witness, so that for infinitely many $i$ there is $x_{i} \in g^{\prime \prime}\left(c_{i} \cup\left\{\alpha_{i}, \beta_{i}\right\}\right)$ such that $c_{i} \in x_{i} \Leftrightarrow x_{i} \notin \mathscr{F}\left(\alpha_{i}, \beta_{i}\right)$. We may assume wlog that this equivalence holds for all $i$. Since $g \in X$, there is a weak support $d$ such that $g \in h^{\prime \prime} d$, and hence $x_{i} \in h^{\prime \prime}\left(c_{i} \cup d \cup\left\{\alpha_{i}, \beta_{i}\right\}\right)$. The assumption $\bigcup c_{i}=\bigcup \alpha_{i}$ implies that $d \cap\left[c_{i}, \alpha_{i}\right)=\varnothing$ for all but finitely many $i$, but then Lemma 2.4 implies that $c_{i} \in x_{i} \Leftrightarrow x_{i} \in \mathscr{F}\left(\alpha_{i}, \beta_{i}\right)$ for all but finitely many $i$. This contradiction completes the proof of clause (ii)(d) of Definition 2.5.

The argument above used the fact that $\mathscr{F}\left(\alpha_{i}, \beta_{i}\right)=\mathscr{F}^{m}\left(\alpha_{i}, \beta_{i}\right)$ and hence is valid so long as $\alpha_{i}<\kappa$ or $\alpha_{i}=\kappa$ and $o(\kappa)<\kappa^{+}$.

We now prove clause (ii) of Lemma 2.6. Assume that $\vec{c}$ is an indiscernible sequence for $(\vec{\alpha}, \vec{\beta})$ and that $\vec{c}$ and $\vec{\alpha}$ are in $X$. First we show that $c_{i}$ is an indiscernible and that $\alpha^{m}\left(c_{i}\right)=\alpha^{m}\left(\alpha_{i}\right)$ for all but finitely many $i$. (Recall that $\alpha^{m}(\alpha)=\alpha$ if $\alpha$ is not an indiscernible.) Note that $\alpha^{m}\left(c_{i}\right) \geq \alpha_{i}$ for all but finitely many $i$ since $c_{i} \leq \alpha^{m}\left(c_{i}\right) \in h^{m \text { " }}\left(c_{i}\right)$ and $\vec{c}$ is an indiscernible sequence for $(\vec{\alpha}, \vec{\beta})$. Since $c_{i}<\alpha_{i}$ it follows that $\alpha^{m}\left(c_{i}\right) \geq \alpha^{m}\left(\alpha_{i}\right)$ for all but finitely many $i \in \omega$. The other direction will follow if we can show that $\alpha^{m}\left(\alpha_{i}\right) \in h^{m}$ " $c_{i}$ for all but finitely many $\alpha$. To see this let $\nu_{i}$ be the least ordinal $\nu$ such that $\alpha^{m}\left(\alpha_{i}\right) \in h^{\prime \prime} \nu$. Then $\nu_{i}<\alpha_{i}$ since $\alpha^{m}\left(\alpha_{i}\right) \in h^{m \text { " }} \alpha_{i}$, and since there is a function $g \in K(\mathscr{F})$ such that $\nu_{i}=g\left(\alpha_{i}\right)$ for all $i$ the assumption that $\vec{c}$ is an indiscernible sequence for $(\vec{\alpha}, \vec{\beta})$ implies that $\nu_{i}<c_{i}$ for all but finitely many $i \in \omega$.

Now we need to see that $c_{i} \in \mathscr{C}^{X}\left(\alpha_{i}, \beta_{i}\right)$ for almost all $i$. Let us first consider the special case in which $\vec{\beta} \in X$. We consider two subcases. For the first subcase we assume that $\alpha^{m}\left(c_{i}\right)=\alpha_{i}$. Then $\alpha_{i}$ is not an indiscernible and since $\beta_{i} \in X$ it follows that $\beta_{i}$ is in $h^{m \text { " }} \alpha_{i}$. It follows that $\beta_{i}$ is in $h^{m \text { " }} c_{i}$ for almost all $i$ : otherwise define a function $g$ by setting $g(\gamma)$ equal to the least ordinal $\nu$ such that $\gamma \in h^{m \text { " }} \nu$. Then the function $g$ is in $K(\mathscr{F})$, but $g\left(\beta_{i}\right)$ is strictly between $c_{i}$ and $\alpha_{i}$ for infinitely many $i$, contradicting the assumption that $\vec{c}$ is an indiscernible sequence for $(\vec{\alpha}, \vec{\beta})$. Since $\beta^{m}\left(c_{i}\right) \in h^{m \text { "s }} c_{i}$ as well, $\beta_{i} \neq \beta^{m}\left(c_{i}\right)$ would imply that the least set $x$ in $\mathscr{F}\left(\alpha_{i}, \beta_{i}\right) \backslash \mathscr{F}\left(\alpha_{i}, \beta^{m}\left(c_{i}\right)\right)$ is in $h^{m \text { "s }} c_{i}$, so that with finitely many exeptions $c_{i}$ must be a member of both $x$ and $\alpha_{i} \backslash x$. This contradiction shows that $\beta_{i}=\beta^{m}\left(c_{i}\right)$, and $c_{i} \in \mathscr{C}^{X}\left(\alpha_{i}, \beta_{i}\right)$, as required. The other subcase, $\alpha^{m}\left(c_{i}\right)=\alpha^{m}\left(\alpha_{i}\right)>\alpha_{i}$, is similar but slightly more complicated. There are $\gamma_{i}$ such that $\beta_{i}=\mathfrak{C}^{m}\left(\alpha^{m}\left(c_{i}\right), \gamma_{i}, \beta^{m}\left(\alpha_{i}\right)\right)\left(\alpha_{i}\right)$. The ordinal $\gamma_{i}$ is given from the ordinals $\beta_{i}$ and $\alpha_{i}$ by a function in $K(\mathscr{F})$, 
and $\gamma_{i} \in h^{m \text { "s }} \alpha_{i}$, so the least $\nu$ such that $\gamma_{i} \in h^{m \text { " }} \nu$ must be below $c_{i}$ for almost all $i$. If $\beta^{m}\left(c_{i}\right) \neq \gamma_{i}$ then the least $x$ such that

$$
x \in \mathscr{F}^{m}\left(\alpha^{m}\left(c_{i}\right), \beta^{m}\left(c_{i}\right)\right) \backslash \mathscr{F}^{m}\left(\alpha^{m}\left(c_{i}\right), \gamma_{i}\right)
$$

is in $h^{m \text { "s }} c_{i}$ and so $c_{i}$ is again in both $x$ and $\alpha_{i} \backslash x$ for almost all $i$. The contradiction shows that $\beta^{m}\left(c_{i}\right)=\gamma_{i}$. To complete the proof that $c_{i} \in \mathscr{C}^{X}\left(\alpha_{i}, \beta_{i}\right)$ we must verify that $\beta^{m}\left(\alpha_{i}\right) \in h^{m \text { "c }} c_{i}$ for almost all $i$. Now $\beta^{m}\left(\alpha_{i}\right) \in h^{m \text { "c }}\left(\alpha_{i}\right)$, so if $g(\xi)$ is the least ordinal $\nu$ such that $\xi \in h^{m \text { "s }} \nu$ then $g$ is in $K(\mathscr{F})$ and $g\left(\beta^{m}\left(\alpha_{i}\right)\right)<\alpha_{i}$ for all $i$. Since $\beta^{m}$ is also in $K(\mathscr{F})$ it follows that $g\left(\beta^{m}\left(\alpha_{i}\right)\right)<c_{i}$ for almost all $i$. This completes the proof for the case when $\vec{\beta}$ is in $X$.

Now let $\vec{\beta}$ be an arbitrary sequence such that $\vec{c}$ is an indiscernible sequence for $(\vec{\alpha}, \vec{\beta})$. By elementarity there must be a sequence $\vec{\beta}^{\prime}$ in $X$ such that $\vec{c}$ is an indiscernible sequence for $\left(\vec{\alpha}, \vec{\beta}^{\prime}\right)$, and by the last paragraph $c_{i} \in \mathscr{C}^{X}\left(\alpha_{i}, \beta_{i}^{\prime}\right)$ for almost all $i$. If $\beta_{i} \neq \beta_{i}^{\prime}$ for infinitely many $i$ then $\vec{c}$ is an indiscernible sequence for two different sequences $\vec{\beta}$ and $\vec{\beta}^{\prime}$, and by elementarity there is a second sequence $\beta^{\prime \prime}$ in $X$ so that $\vec{c}$ is an indiscernible sequence for $\left(\vec{\alpha}, \vec{\beta}^{\prime \prime}\right)$ and $\beta_{i}^{\prime \prime} \neq \beta_{i}^{\prime}$. for infinitely many $i$. This is impossible since, again by the last paragraph, we would also have $c_{i} \in \mathscr{C}^{X}\left(\alpha_{i}, \beta^{\prime \prime}\right)$ for almost all $i$.

2.7 Corollary. Suppose that $\vec{c}, \vec{\alpha}$ and $\vec{\beta}$ are sequences such that $c_{i} \in \mathscr{C}^{X}\left(\alpha_{i}, \beta_{i}\right)$ for all $i \in \omega, \bigcup_{i} c_{i}=\bigcup_{i} \alpha_{i}$, and either $\alpha_{i}<\kappa$ or $o(\kappa)<\kappa^{+}$. Then for any $X^{\prime} \supset X$ we have $c_{i} \in \mathscr{C}^{X^{\prime}}\left(\alpha_{i}, \beta_{i}\right)$ for all but finitely many $i$.

Proof. By applying Lemma 2.6(i) to $\mathscr{C}^{X}$ we get that $\vec{c}$ is an indiscernible sequence for $(\vec{\alpha}, \vec{\beta})$, and by applying Lemma 2.6(ii) to $\mathscr{C}^{X^{\prime}}$ we then get that $c_{i} \in \mathscr{C}^{X^{\prime}}\left(\alpha_{i}, \beta_{i}\right)$ for all but finitely many $i$.

The next corollary may be regarded as a semicontinuity property for the function $\beta(c)$. Note that it is trivial if $X$ is not cofinal in $c$. We conjecture that it is false if "bounded in $c$ " is replaced by "bounded in $c \cap X$ ".

2.8 Corollary. For all but finitely many indiscernibles $c$, if $\alpha$ and $\beta$ are any ordinals such that $c \in \mathscr{C}(\alpha, \beta)$ then $\bigcup_{\beta^{\prime} \geq \beta} \mathscr{C}^{X}\left(\alpha, \beta^{\prime}\right)$ is bounded in $c$. Thus, except at these finitely many exceptional points,

$$
\beta(c) \geq \limsup _{c^{\prime} \rightarrow c}\left\{\beta\left(c^{\prime}\right)+1: c^{\prime}<c \text { and } \alpha\left(c^{\prime}\right)=\alpha(c)\right\}
$$

where the limsup is taken to be 0 if the set of relevant ordinals $c^{\prime}$ is bounded in $c$.

Proof. Suppose that the lemma is false, and let $\vec{c}, \vec{\alpha}$, and $\vec{\beta}$ be $\omega$-sequences such that that for each $i \in \omega$ we have $c_{i} \in \mathscr{C}^{X}\left(\alpha_{i}, \beta_{i}\right)$ but $\bigcup_{\eta \geq \beta_{i}} \mathscr{C}^{X}\left(\alpha_{i}, \eta\right)$ is unbounded in $c_{i}$. By Lemma 2.6(i) and Definition 2.5(ii)(c) each $c_{i}$ must have uncountable cofinality, and we may assume that $\vec{c}$ is strictly increasing.

We first show that we can assume that $\bigcup_{i} c_{i}=\bigcup_{i} \alpha_{i}$. This will involve two stages, the first of which is to show that we can assume that $\alpha_{i}=\alpha\left(c_{i}\right)$ and $\beta_{i}=\beta\left(c_{i}\right)$. Since $c_{i} \in \mathscr{C}\left(\alpha_{i}, \beta_{i}\right)$ there is $\delta_{i}<c_{i}$ such that $\beta\left(\alpha_{i}\right)$ is in $h^{\prime \prime} \delta_{i}$. Then $\bigcup_{\lambda \geq \beta_{i}} \mathscr{E}\left(\alpha_{i}, \lambda\right) \subset \bigcup_{\lambda \geq \beta\left(c_{i}\right)} \mathscr{C}\left(\alpha\left(c_{i}\right), \lambda\right)$, since if $\lambda^{\prime} \in X \cap o\left(\alpha_{i}\right)$ then there is $\lambda<\beta\left(\alpha_{i}\right)$ such that $\lambda^{\prime}=\mathfrak{C}\left(\alpha, \lambda, \beta\left(\alpha_{i}\right)\right)\left(\alpha_{i}\right)$ and $\mathscr{C}\left(\alpha_{i}, \lambda^{\prime}\right)=\mathscr{C}\left(\alpha\left(c_{i}\right), \lambda\right) \backslash \xi$ 
for some $\xi<\alpha_{i}$. It follows that the corollary is also false for $\alpha_{i}=\alpha\left(c_{i}\right)$ and $\beta_{i}=\beta\left(c_{i}\right)$.

If there is an infinite subset $I \subset \omega$ such that $\alpha\left(c_{i}\right)<\alpha\left(c_{i^{\prime}}\right)$ for $i<i^{\prime}$ in $I$ then $c_{i} \leq \alpha\left(c_{i}\right)<c_{i^{\prime}}$ for $i<i^{\prime}$ in $I$ since $\alpha\left(c_{i}\right) \in h^{\prime \prime} c_{i}$, and hence $\bigcup_{i \in I} \alpha\left(c_{i}\right)=\bigcup_{i \in I} c_{i}$. Thus we can assume that there is no such set $I$ and hence that there is an ordinal $\alpha$ such that $\alpha\left(c_{i}\right)=\alpha$ for all sufficiently large $i<\omega$. Set $c=\bigcup_{i \in \omega} c_{i}$. Then $\alpha(c) \leq \alpha$ since $\alpha=\alpha\left(c_{i}\right) \in h^{\prime \prime} c_{i} \subset h^{\prime \prime} c$ for all $i$, and $\alpha \leq \alpha(c)$ since $\alpha(c) \in h^{\prime \prime} c_{i}$ for all sufficiently large $i \in \omega$. Thus we can assume wlog that $\alpha(c)=\alpha\left(c_{i}\right)=\alpha$ for all $i$. Since $\operatorname{cf}(c)=\omega$ the corollary is true for $c$ and hence $\beta(c)>\beta\left(c_{i}\right)$ for all sufficiently large $i<\omega$. Assume wlog that this is true for all $i$, and in addition there is $\xi<c_{0}$ such that $\beta(c) \in h^{\text {" }} \xi$ and $\bigcup_{\beta^{\prime} \geq \beta(c)} \mathscr{C}^{X}\left(\alpha, \beta^{\prime}\right) \cap c$ is contained in $\xi$. Now define new sequences $\vec{\alpha}^{\prime}$ and $\vec{\beta}^{\prime}$ by setting $\alpha_{i}^{\prime}=c$ and $\beta_{i}^{\prime}=\mathfrak{C}\left(\alpha, \beta_{i}, \beta(c)\right)(c)$. If $\xi<\nu<c_{i}$ and $\nu \in \mathscr{C}(\alpha, \eta)$ where $\eta \geq \beta_{i}$ then $\eta<\beta^{m}(c)$ and $\nu \in \mathscr{C}\left(c, \eta^{\prime}\right)$ where $\eta^{\prime}=$ $\mathfrak{C}(\alpha(c), \eta, \beta(c))(c)>\beta_{i}^{\prime}$. Hence $\bigcup_{\eta \geq \beta_{i}^{\prime}} \mathscr{C}^{X}\left(\alpha_{i}^{\prime}, \eta\right) \cap c_{i}=\bigcup_{\eta \geq \beta_{i}} \mathscr{C}^{X}\left(\alpha_{i}, \eta\right) \cap c_{i}$ is unbounded in $c_{i}$ for all $i \in \omega$. Thus the sequences $\vec{c}, \vec{\alpha}^{\prime}$ and $\vec{\beta}^{\prime}$ form a witness to the failure of the corollary such that $\bigcup_{i} c_{i}=\bigcup_{i} \alpha_{i}^{\prime}$.

Thus we can assume that $\bigcup_{i} \alpha_{i}=\bigcup_{i} c_{i}$. For each $i \in \omega$ we define $U_{i}$ to be the set of all subsets $x$ of $c_{i}$ such that for all sufficiently large $d \in$ $c_{i} \cap \bigcup_{\beta^{\prime} \geq \beta_{i}} \mathscr{C}\left(\alpha_{i}, \beta^{\prime}\right)$ we have

$$
\begin{aligned}
d \in x & \text { if } d \in \mathscr{C}\left(\alpha_{i}, \beta_{i}\right), \\
x \cap d \in \mathscr{C}\left(c_{i}, \mathfrak{C}\left(\alpha_{i}, \beta_{i}, \beta^{\prime}\right)\left(c_{i}\right)\right) & \text { if } d \in \mathscr{C}\left(\alpha_{i}, \beta^{\prime}\right) \text { with } \beta_{i}<\beta^{\prime} .
\end{aligned}
$$

The filter $U_{i}$ is countably complete because $\operatorname{cf}\left(c_{i}\right)>\omega$. It is also normal: otherwise let $f \in K(\mathscr{F})$ be such that $\{\nu: f(\nu)<\nu\} \in U_{i}$. If $d \in \mathscr{C}\left(\alpha_{i}, \beta_{i}\right)$ then set $f^{\prime}(d)=f(d)$, and if $d \in \mathscr{C}\left(\alpha_{i}, \beta^{\prime}\right)$ where $\beta^{\prime}>\beta_{i}$ then set $f^{\prime}(d)=\delta$ where $\{\nu<d: f(\nu)=\delta\} \in \mathscr{F}\left(d, \mathfrak{C}\left(\alpha_{i}, \beta_{i}, \beta^{\prime}\right)(d)\right)$. If $f^{\prime}$ is eventually constant, say $f^{\prime}(d)=\delta$ for sufficiently large $\nu$, then $\{\nu: f(\nu)=\delta\} \in U_{i}$, was was to be shown. If $f^{\prime}$ is not eventually constant then there is an infinite increasing sequence $\vec{d}$ such that $f^{\prime}\left(d_{i}\right)<d_{i}$ for all $i$ and $f^{\prime}\left(d_{i}\right)$ is not eventually constant. Let $d=\bigcup_{i} d_{i}$ so that $\beta^{m}(d) \geq \lim \sup _{i} \beta\left(d_{i}\right)$ by Definition $2.5(\mathrm{ii})(\mathrm{c})$. If $U$ is defined on $d$ as $U_{i}$ was defined on $c_{i}$ then $U=\mathscr{F}\left(d, \mathfrak{C}\left(\alpha_{i}, \beta_{i}, \gamma\right)(d)\right)$ where $d \in \mathscr{C}\left(\alpha_{i}, \gamma\right)$, but this is a contradiction because $f$ witnesses that $U$ is not normal.

Thus $U_{i}=\mathscr{F}\left(c_{i}, \delta_{i}\right)$ for some $\delta_{i}<o\left(c_{i}\right)$. Now take $X^{\prime} \supset X$ such that $\mathscr{C}^{X} \in X^{\prime}$. Then $\delta_{i} \in X^{\prime}$ for all $i$ because $U_{i} \in X^{\prime}$. For all but finitely many $i$ we have $c_{i} \in \mathscr{C}^{X^{\prime}}\left(\alpha_{i}, \beta_{i}\right)$ and hence $\delta_{i}=\mathfrak{C}\left(\alpha_{i}, \beta_{i}^{\prime}, \beta_{i}\right)\left(c_{i}\right)$ for some $\beta_{i}^{\prime} \in h^{X^{\prime} “(}\left(c_{i} \cup\left\{\alpha_{i}\right\}\right) \cap \beta_{i}$. Now let $x_{i}$ be the least set in $K(\mathscr{F})$ which is in $\mathscr{F}\left(\alpha_{i}, \beta_{i}\right) \backslash \mathscr{F}\left(\alpha_{i}, \beta_{i}^{\prime}\right)$. Then $x_{i} \in h^{X^{\prime} \text { “" }}\left(c_{i} \cup\left\{\alpha_{i}\right\}\right)$. It follows that $x_{i} \cap c_{i} \in U_{i}$ by the definition of $U_{i}$, but $x_{i} \cap c_{i} \notin \mathscr{F}\left(c_{i}, \delta_{i}\right)$ because $x_{i} \notin \mathscr{F}\left(\alpha, \beta_{i}^{\prime}\right)$. This contradiction completes the proof of the corollary.

Now we know that $\mathscr{C}^{X}$ is a system of indiscernibles for $K(\mathscr{F})$ rather than merely for $m^{X}$ and that $w \subset X \subset h^{\prime \prime}\left(\rho^{X} \cup \bigcup_{\alpha, \beta} \mathscr{E}(\alpha, \beta)\right)$. In order to prove the SCH we would like to show that we need not consider every system of the form $\mathscr{C}^{X}$, but rather that there is a small standard set of systems such that every set $w$ can be covered by a system in the standard set. To do this we will need to 
come to a more precise understanding of the similarity between different systems $\mathscr{C}^{X}$ and $\mathscr{C}^{X^{\prime}}$ of indiscernibles than was given by Corollary 2.7 . In the process we will replace $h^{\text {“" }}\left(\rho^{X} \cup \bigcup_{\alpha, \beta} \mathscr{C}(\alpha, \beta)\right)$ with a more delicate covering: we will define functions $s^{X}$ and $a^{X}$ and then define $h^{“}\left(\delta ; \mathscr{C}^{X}\right)$ to be the smallest set containing $\delta$ and closed under the functions $s^{X}$ and $a^{X}$. Then we will show that $h^{\prime \prime}\left(\delta \cup \bigcup_{\alpha, \beta} \mathscr{C}^{X}(\alpha, \beta)\right)=h^{\prime \prime}\left(\delta ; \mathscr{C}^{X}\right)$ for all $\delta$, so that $w \subset h^{“ "}\left(\delta ; \mathscr{C}^{X}\right)$. Finally we will show that if $X$ and $X^{\prime}$ are two different sets then $s^{X}$ and $a^{X}$ only differ from $s^{X^{\prime}}$ and $a^{X^{\prime}}$ on a bounded subset of $X \cap X^{\prime}$. In the next two sections this fact will be used to select the set of standard systems $\mathscr{C}^{X}$. In $\S 4$ we will actually use a modification of the present definition of $h(\delta ; \mathscr{C})$, and we will be considering systems $\mathscr{C}$ such that it is not true that there is ordinal $\delta$ such that every indiscernible in $\mathscr{C}$ is in $h^{\text {“ }}(\delta ; \mathscr{C})$. Notice that our definition ensures that $\left|h^{\prime \prime}(\delta ; \mathscr{C})\right|=|\delta|$, regardless of the size of $\mathscr{C}$.

The first of the two functions used to define $h^{\text {" }}(\delta, \mathscr{C})$ is the least indiscernible function: $s^{\mathscr{C}}(\alpha, \beta, \gamma)$ is the least member of $\mathscr{C}(\alpha, \beta)$ above $\gamma$ (this definition is slightly modified below). We will observe that under the assumption that $\{o(\alpha): \alpha<\kappa\}$ is bounded below $\kappa$-that is, under the assumption of Theorem 1.1 for countable cofinality-it is sufficient to close $h^{\text {“ }}(\rho ; \mathscr{C})$ under the functions $h$ and $s^{\mathscr{C}}$. For larger sequences we need to introduce the notion of accumulation points; the second function, $a^{X}$, used in the definition of $h^{\prime \prime}\left(\delta ; \mathscr{C}^{X}\right)$ is the least accumulation point function.

It should be noted that the definition of an accumulation point depends directly on the set $X$ as well as on the system $\mathscr{C}^{X}$ of indiscernibles. In particular, the domain of $\mathscr{C}^{X}$ is contained in that of $\mathscr{F}^{m}$, while the ordinals $\beta$ in the definition of an accumulation point are in $X$ and hence come from the domain of $\mathscr{F}$. Thus the definition of accumulation point for $(\alpha, \beta)$ only makes sense if either $\alpha<\kappa$ or $o(\kappa)<\kappa^{+}$, so that $\mathscr{F}^{m}$ and $\mathscr{F}$ agree at $\alpha$.

2.9 Definition. (1) An ordinal $c$ is an accumulation point for $(\alpha, \beta)$ in $X$ if $c, \alpha$ and $\beta$ are in $X, \beta \leq o(\alpha)$, and for all $\beta^{\prime} \in \beta \cap X$ the set

$$
\bigcup\left\{\mathscr{C}^{X}\left(\alpha, \beta^{\prime \prime}\right): \beta^{\prime \prime} \in X \text { and } \beta^{\prime} \leq \beta^{\prime \prime}<\beta\right\}
$$

is cofinal in $X \cap c$. If $\alpha=\alpha(c)$ then this may be stated equivalently

$$
\limsup _{d \rightarrow c^{\prime}}\{\beta(d): \alpha(d)=\alpha \text { and } \beta(d)<\beta\}=\sup (\beta \cap X) .
$$

where $c^{\prime}=\sup (c \cap X)$.

(2) We write $s$ for the least indiscernible function: $c=s(\alpha, \beta, \gamma)$ iff $\gamma<c \in$ $\mathscr{C}(\alpha, \beta)$ and $\bigcup_{\beta^{\prime} \geq \beta} \mathscr{C}\left(\alpha, \beta^{\prime}\right) \cap c \subset \gamma+1$.

(3) We write $a$ for the least accumulation point function: $c=a(\alpha, \beta, \gamma)$ if $c>\gamma, c$ is the least accumulation point for $(\alpha, \beta)$ above $\gamma$, and $\bigcup_{\beta^{\prime} \geq \beta} \mathscr{C}\left(\alpha, \beta^{\prime}\right)$ $\cap c \subset \gamma+1$.

(4) $A$ finite sequence $d$ of ordinals is a (full) support in $X$ if for each $i<$ length $(d)$ there is $(\alpha, \beta) \in h^{\prime \prime}\left(d\lceil i)\right.$ such that either $d_{i}=s\left(\alpha, \beta, d_{i-1}\right)$ or $d_{i}=a\left(\alpha, \beta, d_{i-1}\right)$, where for $i=0$ we take $d_{-1}=0$. The sequence $d$ is $a$ support for a set $e$ of ordinals if $d$ is a support and every member $\nu$ of $e$ is either in $d$ or in $h^{\prime \prime}(d \cap \nu)$.

2.10 Lemma. Assume that $c \in \mathscr{C}(\alpha, \beta)$ and either $\alpha<\kappa$ or $o(\kappa)<\kappa^{+}$. 
Then there is $\gamma \in c \cap X$ such that either $c=s(\alpha, \beta, \gamma)$ or there is an $\eta$ in $h^{\prime \prime}(\{\alpha\} \cup c \cap X)$ such that $c=a(\alpha, \eta, \gamma)$.

Proof. Assume that $c \in \mathscr{C}(\alpha, \beta)$ and $c \neq s(\alpha, \beta, \gamma)$ for any $\gamma \in X \cap c$, and let $\eta \leq o(\alpha)$ be the least member of $X$ such that $\bigcup_{\lambda \geq \eta} \mathscr{E}(\alpha, \lambda)$ is bounded in $X \cap c$. Then $c$ is an accumulation point in $X$ for $(\alpha, \eta)$. First we will show that there is $\gamma \in X \cap c$ such that $c=a(\alpha, \eta, \gamma)$, and then we will show that $\eta \in h^{\prime \prime}(\{\alpha\} \cup c \cap X)$.

Define an $\omega$ sequence $\vec{\alpha}=\left(\alpha_{i}: i \in \omega\right)$ of ordinals as follows: Set $\nu=$ $\bigcup_{\lambda \geq \eta} \mathscr{C}^{X}(\alpha, \lambda) \cap c$, so that $\nu<c$ and $X \cap(c \backslash \nu) \neq \varnothing$ by the choice of $\eta$. Now pick $\alpha_{0} \in X \cap(c \backslash \nu)$ and for $i>0$ define $\alpha_{i}=a\left(\alpha, \eta, \alpha_{i-1}\right)$. If $\alpha_{i}=c$ for some $i$ then we can take $\gamma=\alpha_{i-1}$, since $\alpha_{i^{\prime}} \in X$ for each $i^{\prime}<i$. Otherwise set $\alpha^{\prime}=\bigcup_{i} \alpha_{i} \leq c$. Then Definition 2.5(ii)(c) implies that $\alpha^{\prime} \in \mathscr{C}^{X}(\alpha, \tau)$ for some $\tau \geq \eta$. If $\alpha^{\prime}<c$ then this contradicts the choice of $\alpha_{0}$, while if $\alpha^{\prime}=c$ then $\tau=\beta$ and $c=s\left(\alpha, \beta, \alpha_{0}\right)$, contrary to assumption.

Now we have to show that $\eta \in h^{\prime \prime}(\{\alpha\} \cup X \cap c)$. As a first step we will show that there is an ordinal $\nu \in X \cap c$ such that $h^{\prime \prime}(\{\alpha\} \cup \nu)$ is cofinal in $\eta \cap X$. We define an infinite sequence of indiscernibles: Pick $\nu_{0} \in X \cap c$ so that $\nu_{0} \geq \sup \left(c \cap \bigcup_{\lambda \geq \eta} \mathscr{C}(\alpha, \lambda)\right)$. Now suppose that $\nu_{i}$ is defined. If $h^{\prime \prime}\{\alpha\} \cup \nu_{i}$ is cofinal in $X \cap \eta$ then $\nu_{i}$ is the desired ordinal $\nu$; otherwise let $\nu_{i+1}$ be the least ordinal such that $\nu_{i+1} \in \mathscr{C}\left(\alpha, \lambda_{i+1}\right)$ for some $\lambda_{i+1} \geq \sup \left(h^{\prime \prime}\{\alpha\} \cup \nu_{i}\right)$. Then $\nu_{i+1}<c$ by the choice of $\eta$. This process must stop in finitely many steps, for otherwise let $\nu=\bigcup_{i} \nu_{i}$. Then Corollary 2.8 implies that $\nu \in \mathscr{C}(\alpha, \lambda)$ for some $\lambda$ such that $\lambda>\lambda_{i}$ for all sufficiently large $i<\omega$ and since $\lambda \in h^{\prime \prime}\left(\{\alpha\} \cup \nu_{i}\right)$ for some $i<\omega$ this contradicts the definition of $\nu_{i+1}$.

Now Lemma 2.3 implies that there is a partial function $h^{\xi} \subset h$ such that $h^{\xi} \in h^{\prime \prime} \rho^{X}$ and $\eta \in h^{\xi \text { “ }}(\alpha+1)$. Let $\eta^{\prime}$ be the least member of $h^{\text {“ }}(\{\alpha\} \cup X \cap \nu)$ above $\eta$ and set $f(\gamma)=h^{\xi}(\alpha, \gamma)$ if $h^{\xi}(\alpha, \gamma)<\eta^{\prime}$ and $f(\gamma)=0$ otherwise. Then $f \in h^{\prime \prime}\left(\{\alpha\} \cup\left(X \cap \nu^{\prime}\right)\right)$ for some $\nu^{\prime}<c$. We claim that $f^{\prime \prime} c$ is cofinal in $\eta \cap X$ : If not then there is an ordinal $\zeta \in h^{\prime \prime}(\{\alpha\} \cup c \cap X)$ such that $\zeta<\eta$ and $f^{\prime \prime} c \subset \zeta$. Then the least ordinal $\delta$ such that $f(\delta)>\zeta$ is in $h^{\prime \prime}(\{\alpha\} \cup c \cap X)$, but this is impossible because $c \leq \delta<\alpha$ and $c$ is an indiscernible for a measure on $\alpha$.

Finally, we claim that there is $\nu^{\prime} \in c \cap X$ such that $f^{\prime \prime} \nu^{\prime}$ is cofinal in $X \cap \eta$. This will complete the proof of the lemma, since then $\eta=\sup f^{\prime \prime} \nu^{\prime}$ is in $h^{\prime \prime}(\{\alpha\} \cup X \cap c)$. Define $g: \nu \rightarrow c$ by setting $g(\zeta)$ equal to the least ordinal $\gamma<c$ such that $f(\gamma) \geq h(\alpha, \zeta)$, and set $\nu^{\prime}=\sup$ range $(g)$. Then $\nu^{\prime}<c$ since $g$ witnesses that $\operatorname{cf}\left(\nu^{\prime}\right)=\nu$ while $c$ is regular in $K(\mathscr{F})$, and $f^{\prime \prime} \nu^{\prime}$ is cofinal in $X \cap \eta$ since $h^{\prime \prime}(\nu \cup\{\alpha\})$ is cofinal in $X \cap \eta$.

The next lemma will be used directly in $\S 4$. For $\S 3$ we will have to extend the proof to deal with the case $o(\kappa) \geq \kappa^{+}$, where accumulation points are not defined at $\kappa$.

2.11 Lemma. If $o(\kappa)<\kappa^{+}$then every finite set $e$ of ordinals in $X \cap K(\mathscr{F}) \cap H_{\kappa}$ has a support in $X$.

Proof. We will prove the lemma by induction on $\zeta=\sup e$. If $\zeta$ is not an indiscernible then there is $\gamma \in X \cap \zeta$ such that $\zeta=h(\gamma)$. By the induction hypothesis there is a support $d$ for $\{\gamma\} \cup e \cap \zeta$, and then $d$ is also a support 
for $e$. If $\zeta$ is an indiscernible then by Lemma 2.10 there are $\alpha$ and $\beta$ in $h^{\text {" }} \zeta$ and and $\gamma \in X \cap \zeta$ so that either $\zeta=s(\alpha, \beta, \gamma)$ or $\zeta=a(\alpha, \beta, \gamma)$. By the induction hypothesis there is a support $d$ for $e \cap \zeta \cup\{\gamma\}$ such that $\alpha$ and $\beta$ are in $h^{\prime \prime} d$. Then $d$ is a support for $e$.

Gitik [G?] has shown that this lemma cannot be strengthened by removing the accumulation point function, $a(\alpha, \beta, \gamma)$, from the definition of a support. Gitik's construction requires a model in which there is a measurable cardinal $\kappa$ such that $\{o(\alpha): \alpha<\kappa\}$ is unbounded in $\kappa$. It is known that a cardinal such that $\{o(\alpha): \alpha<\kappa\}$ is unbounded in $\kappa$ is required for the existence of accumulation points, but it is not known whether the measurability of $\kappa$ can be eliminated from the hypothesis to Gitik's result, nor is it known whether a limit of accumulation points can be singular in $K(\mathscr{F})$. An affirmative answer to the following problem would answer both of these questions affirimatively.

2.12 Problem. Suppose that $\alpha=\left(\alpha_{i}: i \in \omega\right)$ is an increasing sequence of measurable cardinals such that $o\left(\alpha_{i+1}\right)=\alpha_{i}$. Is there a larger model $M$ in which each $\alpha_{i}$ is still measurable and such that if $\vec{\gamma}$ and $\vec{\beta}$ are any sequences such that $\gamma_{i}<\alpha_{i}$ and $\beta_{i}<o\left(\alpha_{i}\right)$ for all $i \in \omega$, then there is a sequence $\vec{c}$ which is an indiscernible sequence for $(\vec{\alpha}, \vec{\beta})$ such that $\gamma_{i}<c_{i}$ for all $i \in \omega$ ?

If the answer is yes, then work in $L(\mathscr{U})$, with the ordinals $\alpha_{i}$ as in the hypothesis, and let $M \supset L(\mathscr{U})$ be the model asked for. Let $U_{i}$ be a measure on $\alpha_{i}$ in $M$, and let $j: M \rightarrow N$ be the iterated ultrapower obtained by using each of the measures $U_{i}$ once. The model $N[\vec{\alpha}]$ is a generic extension of $N$ by a variant of Prikry forcing, and hence has the same core model as $N$. Let $\beta_{i}$ be the unique ordinal $\beta<o^{\mathscr{U}}\left(\alpha_{i}\right)$ such that $U_{i} \supset \mathscr{U}\left(\alpha_{i}, \beta\right)$, and in $N[\vec{\alpha}]$ take $X \prec H_{\kappa^{++}}$, where $\kappa=\bigcup_{i} \alpha_{i}$, with $\vec{\alpha}, j(\vec{\alpha})$, and $j(\vec{\beta})$ in $X$. Since $\vec{\alpha}$ is an indiscernible sequence for $(j(\vec{\alpha}), j(\vec{\beta}))$ we have $\alpha_{i} \in \mathscr{C}^{X}\left(j\left(\alpha_{i}\right), j\left(\beta_{i}\right)\right)$ for all sufficiently large $i<\omega$. We claim that there is no sequence $\vec{\gamma}$ such that $\alpha_{i}=s\left(j\left(\alpha_{i}\right), j\left(\beta_{i}\right), \gamma_{i}\right)$ for all sufficiently large $i$. Suppose that $\vec{\gamma}$ is such a sequence. Then the model $M$ contains a indiscernible sequence $\vec{c}$ for $(\vec{\alpha}, \vec{\beta})$ such that $\gamma_{i}<c_{i}$ for all $i \in \omega$. It follows that $j(\vec{c})$ is an indiscernible sequence for $(j(\vec{\alpha}), j(\vec{\beta}))$ such that for all $i$ we have $\gamma_{i} \leq j\left(\gamma_{i}\right)<j\left(c_{i}\right)<\alpha_{i}$ and by elementarity $X$ also satisfies that there is such a sequence $\vec{d}$. Thus $s\left(j\left(\alpha_{i}\right), j\left(\beta_{i}\right), \gamma_{i}\right) \leq d_{i}<\alpha_{i}$ for all sufficiently large $i<\omega$.

We now end $\S 2$ by showing that the least indiscernible function and least accumulation point function are, to some degree, uniquely determined.

2.13 Problem. Suppose that $\vec{\gamma}, \vec{\alpha}$ and $\vec{\beta}$ are $\omega$ sequences of ordinals contained in $X \cap X^{\prime}$, that $\vec{\gamma}$ is strictly increasing, that $\bigcup_{i} \gamma_{i}=\bigcup_{i} \alpha_{i}$, and that either $\alpha_{i}<\kappa$ or $o(\kappa)<\kappa^{+}$. Then the following equations hold for all but finitely many $i \in \omega$

$$
\begin{aligned}
& s^{X}\left(\alpha_{i}, \beta_{i}, \gamma_{i}\right)=s^{X^{\prime}}\left(\alpha_{i}, \beta_{i}, \gamma_{i}\right), \\
& a^{X}\left(\alpha_{i}, \beta_{i}, \gamma_{i}\right)=a^{X^{\prime}}\left(\alpha_{i}, \beta_{i}, \gamma_{i}\right)
\end{aligned}
$$

where the equals sign means that if either side exists then both sides exist and are equal. 
Proof. Consider equation (1). We can assume wlog that $c_{i}=s^{X}\left(\alpha_{i}, \beta_{i}, \gamma_{i}\right)$ exists for all $i \in \omega$. Let $\phi(\vec{c}, \vec{\alpha}, \vec{\beta}, \vec{\gamma})$ be a formula asserting that

The sequence $\vec{c}$ is an indiscernible sequence for $(\vec{\alpha}, \vec{\beta})$ such that $c_{i}>\gamma_{i}$ for all $i<\omega$, and there is no infinite subset $I \subset \omega$ and sequences $\vec{e}=\left(e_{i}: i \in I\right)$ and $\vec{\beta}^{\prime}=\left(\beta_{i}^{\prime}: i \in I\right)$ such that $\gamma_{i}<e_{i}<$ $c_{i}$ and $\beta_{i} \leq \beta_{i}^{\prime}$ for each $i \in I$ and $\vec{e}$ is an indiscernible sequence for $\left(\vec{\alpha}, \vec{\beta}^{\prime}\right)$.

Then $\phi(\vec{c}, \vec{\alpha}, \vec{\beta}, \vec{\gamma})$ is true in $X$ and hence in $V$. Thus $X^{\prime}$ also satisfies that there is a sequence $\vec{d}$ satisfying $\phi(\vec{d}, \vec{\alpha}, \vec{\beta}, \vec{\gamma})$. But then $\phi(\vec{d}, \vec{\alpha}, \vec{\beta}, \vec{\gamma})$ is also true in $V$, and this implies that $\vec{c}$ and $\vec{d}$ are eventually equal.

Now consider equation (2). Assume that $c_{i}=a^{X}\left(\alpha_{i}, \beta_{i}, \gamma_{i}\right)$ exists for all $i \in$ $\omega$. Let $\phi(\vec{d}, \vec{\alpha}, \vec{\beta})$ be a formula asserting that $\vec{d}$ is a sequence of accumulation points for $(\vec{\alpha}, \vec{\beta})$, that is (using Lemma 2.6),

For any sequences $\vec{\nu}$ and $\vec{\lambda}$ such that $\nu_{i}<d_{i}$ and $\lambda_{i}<\beta_{i}$ for all $i \in \omega$ there are sequences $\vec{e}$ and $\vec{\beta}^{\prime}$ such that $\nu_{i}<e_{i}<d_{i}$, $\lambda_{i} \leq \beta_{i^{\prime}}^{\prime}<\beta_{i}$, and $\vec{e}$ is an indiscernible sequence for $\left(\vec{\alpha}, \vec{\beta}^{\prime}\right)$.

Then $X$ satisfies the formula $\theta(\vec{c}, \vec{\alpha}, \vec{\beta}, \vec{\gamma})$ :

$\phi(\vec{c}, \vec{\alpha}, \vec{\beta})$ is true, but $\phi\left(\vec{e}, \vec{\alpha}, \vec{\beta}^{\prime}\right)$ is false for all pairs $\vec{e}$ and $\vec{\beta}^{\prime}$ of sequences defined on an infinite subset $I$ of $\omega$ such that $\gamma_{i}<e_{i}<c_{i}$ and $\beta_{i}^{\prime}>\beta_{i}$ for all $i \in I$, and there are no sequences $\vec{e}$ and $\vec{\beta}^{\prime}$ defined on an infinite subset $I \subset \omega$ such that $\vec{e}$ is an indiscernible sequence for $\left(\vec{\alpha}, \vec{\beta}^{\prime}\right)$ and for all $i \in I$ we have $\gamma_{i}<e_{i} \leq c_{i}$ and $\beta_{i} \leq \beta_{i}^{\prime}$.

Then as in the argument for equation (1), $V$ also satisfies $\theta(\vec{c}, \vec{\alpha}, \vec{\beta}, \vec{\gamma})$ and hence $X^{\prime}$ satisfies that there is a sequence $\vec{d}$ such that $\theta(\vec{d}, \vec{\alpha}, \vec{\beta}, \vec{\gamma})$. Then $\theta(\vec{d}, \vec{\alpha}, \vec{\beta}, \vec{\gamma})$ is also true in $V$, and it follows that $\vec{c}$ and $\vec{d}$ must be equal except on an initial segment.

2.14 Corollary. For any $X$ and $X^{\prime}$ there is an ordinal $\xi<\kappa$ such that whenever $\alpha, \beta, \gamma \in X \cap X^{\prime}, \gamma>\xi$, and $\alpha<\kappa$ we have $s^{X}(\alpha, \beta, \gamma)=s^{X^{\prime}}(\alpha, \beta, \gamma)$ and $a^{X}(\alpha, \beta, \gamma)=a^{X^{\prime}}(\alpha, \beta, \gamma)$.

Proof. If the corollary is false then there would be sequences $\vec{\alpha}, \vec{\beta}$ and $\vec{\gamma}$ such that for each $i \in \omega$ we have $\gamma_{i}<\alpha_{i} \leq \gamma_{i+1}$ and the corollary is false for $\alpha_{i}$, $\beta_{i}$ and $\gamma_{i}$, contradicting Lemma 2.13 .

\section{UNCOUNTABLE COFINALITY}

In this section we prove Theorem 1.1(ii), which is the easier part of the main theorem. Most of the necessary tools are in $\S 2$; the only difficulty is the indiscernibles for measures on $\kappa$, which are not covered by Lemma 2.10 and Corollary 2.14. We assume throughout this section that $\kappa$ is a singular strong limit cardinal of uncountable cofinality.

For each function $h: \kappa \rightarrow H_{\kappa}$ in $K(\mathscr{F})$ and each ordinal $\alpha<\kappa$ pick a set $X=X^{h, \alpha} \prec H_{\kappa^{++}}$with $|X|<\kappa$ such that $\alpha \cup\{h, \alpha\} \subset X$ and ${ }^{\omega} X \subset X$. The 
main lemma of this section is

3.1 Lemma. For every set $w \subset \kappa$ of cardinality less than $\kappa$ there is a function $h$ and a sequence $\vec{\alpha}=\left(\alpha_{k}: k \in \omega\right)$ such that $w \subset \bigcup_{k \in \omega} X^{h, \alpha_{k}}$.

Theorem 1.1(ii) follows easily from Lemma 3.1: Since $K(\mathscr{F})$ satisfies the GCH there are only $\kappa^{+}$many functions $h$, and since $\kappa$ is a strong limit cardinal of uncountable cofinality there are only $\kappa$ many sequences $\vec{\alpha}$. Hence there are only $\kappa^{+}$many pairs $(h, \vec{\alpha})$ and hence only $\kappa^{+}$many sets $\bigcup_{k \in \omega} X^{h, \alpha_{k}}$. Since each of these sets has cardinality less than $\kappa$ and $\kappa$ is a strong limit cardinal, each of these sets has fewer than $\kappa$ subsets and thus there are only $\kappa^{+}$subsets of $\kappa$ of cardinality less than $\kappa$, and hence $2^{\kappa}=\kappa^{+}$.

We need some preliminaries before proving Lemma 3.1. Let $X \prec H_{\kappa^{++}}$be arbitrary. For $\nu<\kappa$ define $A_{\nu}^{X}$ to be $\left\{s(\kappa, \beta, \nu): \beta<o^{m^{X}}(\kappa)\right\}$. Then $A_{\nu}^{X}$ is $\omega$-closed. To see this, suppose that $c$ is a limit point of $A_{\nu}^{X}$ of cofinality $\omega$, say $c=\bigcup_{i} c_{i}$ where $c_{i}=s\left(\kappa, \beta_{i}, \nu\right) \in A_{\nu}^{X}$. Then by Definition 2.5(ii)(c) we have $c \in \mathscr{C}^{X}(\kappa, \beta)$ where $\beta>\beta_{i}$ for all sufficiently large integers $i$. If $c \neq s(\kappa, \beta, \nu)$ then by Definition 2.9(2) it is because there is $\beta^{\prime} \geq \beta$ and $c^{\prime} \in \mathscr{C}\left(\kappa, \beta^{\prime}\right)$ such that $\nu<c^{\prime}<c$, but in this case we would also have $c_{i} \neq s\left(\kappa, \beta_{i}, \nu\right)$ for all $i$ large enough that $c_{i}>c^{\prime}$ and $\beta_{i}<\beta$.

3.2 Lemma. $A_{\nu}^{X}$ is cofinal in $\kappa$ for all sufficiently large ordinals $\nu<\kappa$.

Proof. Suppose that the lemma is false and define a sequence $\left(\nu_{k}: k \in \omega\right)$ of ordinals less than $\kappa$ as follows: set $\nu_{0}=0$ and for $k>0$ let $\nu_{k}$ be the least member of $X \backslash \sup \left(A_{\nu_{k-1}}^{X}\right)$. Let $\alpha=\sup _{k} \nu_{k}$, so that $\alpha<\kappa$ since $\operatorname{cf}(\kappa)>\omega$. Let $\beta_{k} \leq o^{m}(\kappa)$ be the least ordinal $\beta$ such that $\bigcup_{\lambda \geq \beta} \mathscr{C}(\kappa, \lambda) \backslash \nu_{k}=\varnothing$. Then $\beta_{k+1} \leq \beta_{k}$ so there are $\beta$ and $k_{0}$ such that $\beta_{k}=\beta$ for all $k \geq k_{0}$. Then Definition 2.5(ii)(c) implies that $\beta^{m}(\alpha) \geq \beta$, contradicting the definition of $\beta_{k_{0}}$.

For each $X \prec H_{\kappa^{++}}$let $\nu^{X}$ be the least ordinal $\nu$ such that $A_{\nu}^{X}$ is cofinal in $\kappa$ and let $C^{X}=A_{\nu^{X}}^{X}$. We need a notion of supports which is more general than that of Definition 2.9. Fix $\eta^{X}$ in the interval $\nu^{X} \leq \eta^{X}<\kappa$ so that the set

$$
D^{X}=\left\{c \in C^{X}: \operatorname{cf}(c)=\omega \text { and } \beta^{X}(c) \in h^{X “}\left(\eta^{X}\right)\right\}
$$

is stationary. Call a finite sequence $d$ of ordinals an extended $X$-support if for each $d_{i} \in d$ one of the following two cases holds:

(i) There are $\alpha<\kappa$ and $\beta<o(\alpha)$ in $h^{X \text { " }}\left(\eta^{X} \cup d\lceil i)\right.$ such that either $d_{i}=s\left(\alpha, \beta, d_{i-1}\right)$, or $d_{i}=a\left(\alpha, \beta, d_{i-1}\right)$, or

(ii) For every $\alpha \in D^{X} \backslash d_{i}+1$ there is $\beta \in h^{X \text { “" }}\left(d\left\lceil i \cup\{\alpha\} \cup \eta^{X}\right)\right.$ such that either $d_{i}=s\left(\alpha, \beta, d_{i-1}\right)$ or $d_{i}=a\left(\alpha, \beta, d_{i-1}\right)$.

As in the last section, we say that $d$ is an extended $X$-support for $e$ if $d$ is an extended $X$-support and every member $\nu$ of $e$ is either in $d$ or in $h^{X “}\left(\eta^{X} \cup(d \cap \nu)\right)$.

3.3 Lemma. For every finite sequence $e$ of ordinals in $X$ there is a sequence $d$ which is an extended $X$-support for $e$.

Proof. Define $\delta_{\alpha}(\lambda)=\mathfrak{C}^{m}(\kappa, \lambda, \beta(\alpha))(\alpha)$ for $\alpha \in D^{X}$ and $\lambda<\beta(\alpha)$. We will extend the inductive proof of Lemma 2.11 by showing that for each $c \in$ 
$\bigcup_{\beta} \mathscr{C}(\kappa, \beta)$ there are ordinals $\lambda \in h^{\prime \prime} c \cap o^{m}(\kappa)$ and $\gamma<c$ such that either $c=s\left(\alpha, \delta_{\alpha}(\lambda), \gamma\right)$ for all $\alpha \in D^{X} \backslash c+1$ or $c=a\left(\alpha, \delta_{\alpha}(\lambda), \gamma\right)$ for all $\alpha \in$ $D^{X} \backslash c+1$. Since $\lambda \in h^{\prime \prime} c$ there is a finite subset $q$ of $c$ such that $\lambda \in h^{\prime \prime} q$, and since $\beta(\alpha) \in h^{\prime \prime} \eta^{X}$ it follows that $\delta_{\alpha}(\lambda) \in h^{\prime \prime}\left(\eta^{X} \cup q \cup\{\alpha\}\right)$. By the induction hypothesis there is an extended $X$-support $d^{\prime}$ for $q \cup\{\gamma\}$, and then $d=d^{\prime} \cup\{c\}$ is an extended support for $c$, completing the proof of the lemma.

The proof depends on the fact that $\beta(\alpha)<\eta^{X}$ for all $\alpha \in D^{X}$, which implies that $\delta_{\alpha}(\lambda)$ gives an exact correlation between indiscernibles for $\kappa$ and those for ordinals $\alpha \in D^{X}:$ if $\mathscr{C}(\kappa, \lambda) \cap\left[\eta^{X}, \alpha\right) \neq \varnothing$ then $\delta_{\alpha}(\lambda)$ is defined and

$$
\mathscr{C}(\kappa, \lambda) \cap\left[\eta^{X}, \alpha\right)=\mathscr{C}\left(\alpha, \delta_{\alpha}(\lambda)\right) \backslash \eta^{X},
$$

and conversely if $\mathscr{C}(\alpha, \delta) \neq \varnothing$ then there is $\lambda$ such that $\delta=\delta_{\alpha}(\lambda)$ and equation (1) holds.

Now if $\alpha \in D^{X} \backslash c+1$ then $c \in \mathscr{C}\left(\alpha, \delta_{\alpha}(\beta(c))\right)$ and it follows from Lemma 2.10 that for some $\gamma<c$ either $c=s\left(\alpha, \delta_{\alpha}(\beta(c)), \gamma\right)$ or there is $\delta$ such that $c=a(\alpha, \delta, \gamma)$. Suppose first that $c=s\left(\alpha, \delta_{\alpha}(\beta(c)), \gamma\right)$. Then for any $\alpha^{\prime}$ in $D^{X} \backslash c$ we have

$$
\begin{aligned}
\bigcup_{\beta \geq \delta_{\alpha^{\prime}}(\beta(c))} \mathscr{C}\left(\alpha^{\prime}, \beta\right) \cap\left(\eta^{X}, c\right] & =\bigcup_{\beta \geq \beta(c)} \mathscr{C}(\kappa, \beta) \cap\left(\eta^{X}, c\right] \\
& =\bigcup_{\beta \geq \delta_{\alpha}(\beta(c))} \mathscr{C}(\alpha, \beta) \cap\left(\eta^{X}, c\right]
\end{aligned}
$$

and it follows that $c=s\left(\alpha^{\prime}, \delta_{\alpha^{\prime}}(\beta(c)), \gamma\right)$ for all $\alpha^{\prime} \in D^{X} \backslash c+1$, as required.

Thus we can assume that for each $\alpha \in D^{X} \backslash c+1$ there is $\lambda_{\alpha} \in h^{\prime \prime}(c \cup$ $\{\alpha\})$ and $\gamma_{\alpha}$ such that $c=a\left(\alpha, \lambda_{\alpha}, \gamma_{\alpha}\right)$. Since $\lambda_{\alpha} \in h^{\prime \prime}(c \cup\{\alpha\})$, there is $\lambda_{\alpha}^{\prime}$ such that $\lambda_{\alpha}=\delta_{\alpha}\left(\lambda_{\alpha}^{\prime}\right)$. Now $c=a\left(\alpha, \lambda_{\alpha}, \gamma_{\alpha}\right)$ implies that $\bigcup_{\beta \geq \lambda_{a}} \mathscr{C}(\alpha, \beta)$ is bounded in $c$. If $\alpha^{\prime}$ is any other member of $D^{X} \backslash c+1$ then the argument in the last paragraph implies that $\bigcup_{\beta \geq \delta_{\alpha^{\prime}}\left(\lambda_{\alpha}^{\prime}\right)} \mathscr{C}\left(\alpha^{\prime}, \beta\right)$ is bounded in $c$, so that $c$ is not an accumlation point for $\left(\alpha^{\prime}, \beta\right)$ for any $\beta>\delta_{\alpha^{\prime}}\left(\lambda_{\alpha}^{\prime}\right)$. Since $c$ is an accumulation point for $\left(\alpha^{\prime}, \delta_{\alpha^{\prime}}\left(\lambda_{\alpha^{\prime}}^{\prime}\right)\right)$ it follows that $\lambda_{\alpha^{\prime}}^{\prime} \leq \lambda_{\alpha}^{\prime}$, and since $\alpha$ and $\alpha^{\prime}$ were arbitrary it follows that $\lambda_{\alpha}^{\prime}$ does not vary with $\alpha \in D^{X} \backslash c+1$. A similar argument shows that $\gamma_{\alpha}$ also does not vary with $\alpha \in D^{X} \backslash c+1$, and this completes the proof of the lemma.

Proof of Lemma 3.1. Fix $w \subset \kappa$ of cardinality less than $\kappa$, together with a set $X \prec H_{\kappa^{++}}$such that $w \subset X$, and let $h=h^{X}\left\lceil\left\{\nu: h^{X}(\nu) \in H_{\kappa}\right\}\right.$. Define the increasing sequence $\left(\alpha_{i}: i<\omega\right)$ of ordinals below $\kappa$ by induction on $i$ : First set $\alpha_{0}$ equal to $\eta^{X}$. Now suppose that $\alpha_{i}$ has been defined, and set $X_{i}=X^{h, \alpha_{i}}$. By Corollary 2.14 there is an ordinal $\xi_{i}<\kappa$ such that for any ordinals $\alpha, \beta, \gamma \in X \cap X_{i}$ with $\gamma>\xi_{i}$ we have $s^{X}(\alpha, \beta, \gamma)=s^{X_{i}}(\alpha, \beta, \gamma)$ and $a^{X}(\alpha, \beta, \gamma)=a^{X_{i}}(\alpha, \beta, \gamma)$. Let $\alpha_{i+1}$ be an ordinal such that $\alpha_{i+1}>$ $\max \left\{\xi_{i}, \alpha_{i}\right\}$, and $\alpha_{i+1}$ is sufficiently large that $\left\{c \in D^{X} \cap C^{X_{i}}: \beta^{X_{i}}(c) \in\right.$ $\left.h^{X_{i}{ }^{\prime \prime}}\left(\alpha_{i+1}\right)\right\}$ is stationary. We claim that $X \subset \bigcup_{i} X_{i}$. By Lemma 0.0 it is enough to show that every extended $X$-support $d$ is in $\bigcup_{i} X_{i}$. Let $\nu=\sup _{i} \alpha_{i}$ and let $i$ be large enough that $d \cap \nu \subset \alpha_{i}$. We will show that $d \in X_{i}$.

Since $d \cap \nu \subset \alpha_{i} \subset X_{i}$ it is enough to prove by induction on $j$ that $d_{j} \in X_{i}$ for all $j$ with $d_{j} \geq \nu$. Suppose that $d\left\lceil j \in X_{i}\right.$. Now if case (i) of the definition of an extended $X$-support holds for $d_{j}$ then $d_{j}$ is equal to 
either $s^{X}\left(\alpha, \beta, d_{j-1}\right)$ or $a^{X}\left(\alpha, \beta, d_{j-1}\right)$ for some $\alpha$ and $\beta$ in $h^{X \text { “' }}\left(\eta^{X} \cup d\lceil j)\right.$. Since $\eta^{X} \cup d\left\lceil j\right.$ is in $X_{i}$ we know that $\alpha$ and $\beta$ are in $X_{i}$, and hence $s^{X_{i}}\left(\alpha, \beta, d_{j-1}\right)$ is in $X_{i}$. Now $s^{X}(\alpha, \beta, \gamma)=s^{X_{i}}(\alpha, \beta, \gamma)$ (and similarly for $a(\alpha, \beta, \gamma)$ ) for all $\gamma \geq \alpha_{i}$, so we are done unless $d_{j-1}<\alpha_{i}$ in which case $d_{j}=s^{X}\left(\alpha, \beta, d_{j-1}\right)=s^{X}\left(\alpha, \beta, \alpha_{i}\right)=s^{X_{i}}\left(\alpha, \beta, \alpha_{i}\right) \in X_{i}$ (or similarly for $\left.a\left(\alpha, \beta, d_{j-1}\right)\right)$.

Now if, on the other hand, case (ii) holds then $\left\{\alpha \in D^{X}: \beta^{X_{i}}(\alpha) \in h^{X_{i}}{ }^{\prime \prime} \nu\right\}$ is stationary, and hence is unbounded. Thus we can pick $\alpha$ in this set so that $\alpha>d_{i}$ and use the same argument as for case (i).

\section{Countable Cofinality}

The case of cofinality $\omega$ is more delicate than the case of uncountable cofinality. With $\operatorname{cf}(\kappa)>\omega$ every countable sequence $\vec{\alpha}$ of ordinals below $\kappa$ was bounded in $\kappa$. This meant that there were only $\kappa$ many such sequences and hence the theorem could be proved by covering every small subset of $\kappa$ with a union $\bigcup_{k} X^{h, \alpha_{k}}$ of basic covering sets. Since this trick will not work when $\operatorname{cf}(\kappa)=\omega$ we will have to define standard systems of indiscernibles having a stronger maximality property. These systems are given by Lemma 4.3 below. In order to illustrate the method we begin with another result which uses a technique suggested by Jensen to show that under a stronger hypothesis there is system $\mathscr{C}$ which does not depend at all on the set $X$ to be covered, and hence is maximal in the strongest possible sense. This generalizes the result of Jensen and Dodd [D] that if $L(\mu)$ exists but $0^{\dagger}$ does not, and there is a Prikry sequence over $L(\mu)$, then there is a unique (up to finite changes) maximal Prikry sequence over $L(\mu)$.

4.1 Theorem. Assume that $\alpha^{\omega} \leq \alpha^{+}$for all ordinals $\alpha$. If there is no regular limit of measurable cardinals in $K(\mathscr{F})$ and no proper class of measurable cardinals then there is a system $\mathscr{C}$ of indiscernibles for $K(\mathscr{F})$ with the following maximality property: if $\mathscr{C}^{\prime}$ is any other system of indiscernibles for $K(\mathscr{F})$ then $\cup\left\{\mathscr{C}^{\prime}(\xi) \backslash \mathscr{C}(\xi): \xi\right.$ is a cardinal $\}$ is finite.

Proof. Since we are dealing with sequences $\mathscr{F}$ which have at most one measure per cardinal, we will write $\mathscr{F}(\alpha)$ instead of $\mathscr{F}(\alpha, 0)$ and $\mathscr{C}(\alpha)$ instead of $\mathscr{C}(\alpha, 0)$.

We will use recursion over $\alpha$ to construct systems $\mathscr{C}_{\alpha}$ of indiscernibles for $\mathscr{F} \mid \alpha$ which have the desired maximality property. Assume as an induction hypothesis that we have constructed $\mathscr{C}_{\alpha^{\prime}}$ for all $\alpha^{\prime}<\alpha$. The successor case is easy: a maximal system of indiscernibles for $\mathscr{F} \mid \alpha$ is also a maximal system for $\mathscr{F}\lceil(\alpha+1)$ unless $\alpha$ is measurable in $K(\mathscr{F})$, in which case the maximal system $\mathscr{C}_{\alpha+1}$ for $\mathscr{F}\lceil(\alpha+1)$ can be obtained by adding a maximal Prikry sequence for $\mathscr{F}(\alpha)$ to the maximal system $\mathscr{C}_{\alpha}$ for $\mathscr{F}\lceil\alpha$.

If $\alpha$ is a limit ordinal but not a limit of measurable cardinals then there is no problem, so we can assume that $\alpha$ is a limit of measurable cardinals. Then the hypothesis implies that $\alpha$ is singular in $K(\mathscr{F})$, say $\alpha=\sup _{\nu<\lambda} \alpha_{\nu}$ where $\lambda=\operatorname{cf}^{K(\mathscr{F})}(\alpha)$ and $\left(\alpha_{\nu}: \nu<\lambda\right) \in K(\mathscr{F})$ is continuous and unbounded in $\alpha$. Take $X \prec H_{\alpha^{+}}$so that $|X|<\kappa, \lambda \subset X, \alpha \in X$, and $\left(\mathscr{C}_{\xi}: \xi<\alpha\right) \in X$. The required system $\mathscr{C}=\mathscr{C}_{\alpha}$ is obtained by using $\mathscr{C}^{X}$ to combine the systems $\mathscr{C}_{\alpha_{\nu}}$ : 


$$
\mathscr{C}(\zeta)= \begin{cases}\mathscr{C}^{X}(\zeta) & \text { if } \zeta \in X \\ \mathscr{C}_{\alpha_{\nu+1}}(\zeta) & \text { if } \zeta \notin X \text { and } \alpha_{\nu}<\zeta<\alpha_{\nu+1}\end{cases}
$$

We claim that for each $\nu<\lambda$ the restriction $\mathscr{C}^{\prime}=\mathscr{C}\left\lceil\left(\alpha_{\nu+1} \backslash \alpha_{\nu}\right)\right.$ is a member of $X$. Consider the following two sets:

$$
\begin{aligned}
& c_{1}=\bigcup\left\{\mathscr{C}^{X}(\zeta) \backslash \mathscr{C}_{\alpha_{\nu}}(\zeta): \alpha_{\nu}<\zeta \leq \alpha_{\nu+1}\right\}, \\
& c_{2}=\bigcup\left\{\mathscr{C}_{\alpha_{\nu}}(\zeta) \backslash \mathscr{C}^{X}(\zeta): \zeta \in X \text { and } \alpha_{\nu}<\zeta<\alpha_{\nu+1}\right\} .
\end{aligned}
$$

The set $c_{1}$ is finite by the maximality of $\mathscr{C}_{\alpha_{\nu}}$, and since $c_{1} \subset X$ it follows that $c_{1} \in X$. The set $c_{2}$ is finite by Lemma 2.13. It is a subset of $X$ : otherwise let $\nu$ be the largest member of $c_{2} \backslash X$ and and let $\zeta$ be the ordinal such that $\nu \in \mathscr{C}_{\alpha_{\nu}}(\zeta)$. Then $\mathscr{C}_{\alpha_{\nu}}(\zeta) \in X$, and if $\gamma$ is the least member of $X$ above $\nu$ then $\nu$ is the largest member of $\mathscr{C}_{\alpha_{\nu}}(\zeta)$ below $\nu$ and hence is in $X$. It follows that $c_{2} \in X$. Then $\mathscr{C}_{\alpha_{\nu}}, c_{1}$ and $c_{2}$ are all in $X$ and since $\mathscr{C}_{\alpha_{\nu}}$ can be converted to $\mathscr{C}^{\prime}$ by adding $c_{1}$ and deleting $c_{2}$ it follows that $\mathscr{C}^{\prime} \in X$.

Now suppose that $\mathscr{C}$ is not a system of indiscernibles. Then there is a countable set $I \subset \lambda$ such that $\mathscr{C}^{I}=\mathscr{C} \mid \bigcup_{\nu \in I}\left(\alpha_{\nu+1} \backslash \alpha_{\nu}\right)$ is not a system of indiscernibles. Since ${ }^{\omega} X \subset X, \mathscr{C}^{I}$ is in $X$ and so by elementarity it is true in $X$ that $\mathscr{C}^{I}$ is not a system of indiscernibles. This is absurd because $\mathscr{C} \mid X=$ $\mathscr{C}^{X}$. The same argument shows that $\mathscr{C}$ is maximal and completes the proof of the theorem.

A result due to Jensen and independently to myself and P. Matet (see [Mi84a]) shows that the hypothesis of Theorem 4.1 cannot be weakened further: If there is a regular limit $\kappa$ of measurable cardinals or a class of measurable cardinals then there is a model with the same cardinals in which every measurable cardinal has a Prikry sequence, but in which there is no system $\mathscr{C}$ of indiscernibles such that $\mathscr{C}(\alpha) \neq \varnothing$ for unboundedly many $\alpha<\kappa$.

Our proof of Theorem 1.1(i) is based on the same ideas. We will assume that the conclusion of Theorem 1.1(i) is false, that is, that there is an ordinal $\beta_{0}<\kappa$ such that $o(\alpha)<\beta_{0}$ for all $\alpha$, and use this to prove the $\mathrm{SCH}$. Although there is no single maximal system of indiscernibles for $\mathscr{F}$ we will be able to construct a set of standard systems, each of which is a maximal system of indiscernibles on domains determined by some particular function in $K(\mathscr{F})$. We no longer are restricted to one measure per cardinal, but since $o(\alpha)<\alpha$ for all $\alpha$ some simplification of notation is still possible. We write $\mathscr{C}(\alpha)$ for $\bigcup_{\beta<o(\alpha)} \mathscr{C}(\alpha, \beta)$. Note that $\mathscr{C}(\alpha, \beta)=\{\nu \in \mathscr{C}(\alpha): o(\nu)=\beta\}$.

The restriction to cardinals in Theorem 4.1 can be weakened slightly, but it cannot be eliminated. Thus we need to introduce some technical apparatus so that we can restrict our attention to systems $\mathscr{C}$ of indiscernibles such that $\mathscr{C}(\alpha)=\varnothing$ whenever $\alpha$ is not a cardinal in the real world. For simplicity we will further restrict ourselves to systems of indiscernibles such that $\mathscr{C}(\alpha)=\varnothing$ whenever there is $\lambda<\alpha$ such that $\lambda^{\omega} \geq \alpha$. Call an ordinal $\alpha$ full if $\alpha$ is a cardinal and $\lambda^{\omega}<\alpha$ for all $\lambda<\alpha$. In order to cover a set with such restricted systems fix functions $\sigma$ and $\tau$ such that $\sigma(\nu, \cdot):|\nu| \cong \nu$ and $\tau(\nu, \cdot):{ }^{\omega} \nu \cong|\nu|^{\omega}$ for all ordinals $\nu$. For the rest of this section we will assume that all systems of indiscernibles mentioned are empty except on full 
cardinals and that every elementary substructure $X$ of $H_{\kappa^{++}}$which we use contains the set $\{\sigma, \tau\} \cup \beta_{0}$ and also contains all of its limit points of cofinality at most $\beta_{0}$. Notice that by Corollary 2.8 this implies that there are at most finitely many accumulation points in $\mathscr{C}^{X}$, so that we can assume wlog that there are none.

Let $\mathscr{H}$ be the class of $h \in K(\mathscr{F})$ such that there is a ordinal $\eta$ such that $h$ maps a cofinal subset of $\eta$ into $\eta$ so that $h(\nu)>\nu$ for all $\nu \in \operatorname{domain}(h)$. For $h \in \mathscr{H}$ we will abuse notation by writing domain $(h)$ for $\eta$ and, if $\xi<\eta$, by writing $h\left\lceil\xi\right.$ for $h \cap \xi^{2} \in \mathscr{H}$. The following definition is a modification of notation from earlier in the paper:

4.2 Definition. If $h \in \mathscr{H}$ with $\eta=\operatorname{domain}(h)$ and $\mathscr{C}$ is a system of indiscernibles then we write $h^{\prime \prime}(\gamma ; \mathscr{C})$ for the smallest set $Y \subset \eta+1$ such that

(i) $\gamma \cup\{\eta\} \subset Y$ and $Y$ is closed under the canonical pairing function,

(ii) $h^{\prime \prime} Y \subset Y, \sigma^{\prime \prime} Y^{2} \subset Y$, and $\tau^{\text {" }}\left(Y \times[Y]^{\omega}\right) \subset Y$, and

(iii) $s^{\mathscr{C}}\left(\alpha, \beta, \gamma^{\prime}\right) \in Y$ whenever $\alpha, \beta, \gamma^{\prime} \in Y$ and $s^{\mathscr{C}}\left(\alpha, \beta, \gamma^{\prime}\right)<\eta$.

Note that under this definition it is still true that if $h=h^{X} \uparrow\left\{\nu: \nu<h^{X}(\nu)<\right.$ $\kappa\}$ then $X \cap \kappa \subset h^{“}\left(\rho^{X} ; \mathscr{C}^{X}\right)$.

The next lemma asserts the existence of the standard systems of indiscernibles which are used in the proof of Theorem 1.1(i). We will first prove Theorem 1.1(i) under the assumption that Lemma 4.3 holds, and then prove Lemma 4.3.

4.3 Lemma. For all $h \in \mathscr{H}$ there is a system $\mathscr{C}^{h}$ such that for any system $\mathscr{C}$ of indiscernibles of the form $\mathscr{C}^{Y}$, any sufficiently large $\delta<\eta=\operatorname{domain}(h)$, and any $\alpha \in h^{\prime \prime}\left(\delta ; \mathscr{C}^{h}\right)$ we have $\mathscr{C}(\alpha) \backslash \delta \subset \mathscr{C}^{h}(\alpha)$.

The following lemma will be used in the proof of both Theorem 1.1(i) and of Lemma 4.3.

4.4 Lemma. Suppose $X \prec H_{\kappa^{++}}, \mathscr{C}$ is a system of indiscernibles of the form $\mathscr{C}^{Y}, \gamma \in X$, and $\gamma>\rho^{X}$. Then there is a $\delta<\gamma$ such that $\mathscr{C}(\alpha) \backslash \delta \subset \mathscr{C}^{X}(\alpha)$ for all $\alpha \in X \cap(\gamma+1 \backslash \delta)$.

Proof. If either of $X$ or $\bigcup_{\alpha} \mathscr{C}(\alpha)$ is bounded in $\gamma$ then the lemma is immediate. If both are unbounded then by elementarity $X$ satisfies that there is a system $\mathscr{D}$ of indiscernibles which is unbounded in $\gamma$, and all but finitely many of the indisceribles in $\mathscr{D}$ must be indiscernibles in $\mathscr{C}^{X}$ since $c \in h^{X{ }^{\prime \prime}} c$ for every ordinal $c$ which is not an indiscernible in $\mathscr{C}^{X}$. Thus $\bigcup_{\alpha} \mathscr{C}^{X}(\alpha)$ is unbounded in $\gamma$.

Now suppose that $c$ and $\alpha$ are ordinals such that $c \in \mathscr{C}(\alpha) \backslash \mathscr{C}^{X}(\alpha)$. Set $\delta(c)=0$ if $\mathscr{C}^{X}(\alpha) \cap c=\varnothing$ and $\delta(c)=\sup \left(\mathscr{C}^{X}(\alpha) \cap c\right)$ otherwise, and set $\nu(c)=\inf (X \backslash c+1)$. Then $\delta(c)$ and $\nu(c)$ are both in $X$ (for $\delta(c)$, note that $\operatorname{cf}(\delta(c)) \leq \beta_{0}$ by Corollary 2.8 and recall that $X$ contains its limit points of cofinality at most $\left.\beta_{0}\right)$ and $\delta(c)<c<\nu(c)<\gamma$ and $\mathscr{C}^{X}(\alpha) \cap(\nu(c) \backslash \delta(c))=\varnothing$.

Now if the lemma is false then there must exist sequences $\vec{c}, \vec{\alpha}, \vec{\delta}$ and $\vec{\nu}$ in $X$ such that for each $n \in \omega$ we have $c_{n} \in \mathscr{C}\left(\alpha_{n}\right) \backslash \mathscr{C}^{X}(\alpha), \delta_{n}=\delta\left(c_{n}\right)$, $\nu_{n}=\nu\left(c_{n}\right)$ and $\nu_{n} \leq \delta_{n+1}$. Then by elementarity $X$ satisfies that there is a sequence $\vec{d}=\left(d_{n}: n \in \omega\right)$, a system $\mathscr{D}$ of indiscernibles, and a function $h \in K(\mathscr{F})$ such that for each $n$ we have $\delta_{n}<d_{n}<\nu_{n}, d_{n} \in \mathscr{C}\left(\alpha_{n}\right)$, and $\alpha_{n} \in h^{\text {“c }} d_{n}$. Then $d_{n} \in \bigcup_{\alpha} \mathscr{C}^{X}(\alpha)$ for all but finitely many $n \in \omega$, for otherwise 
$d_{n} \in h^{X \text { “ }}\left(d_{n}\right)$ for infinitely many $n$, contradicting the assumption that $\mathscr{D}$ is a system of indiscernibles. Then $\alpha_{n} \in h^{\text {" }} d_{n}$ implies that $\alpha^{X}\left(d_{n}\right) \leq \alpha_{n}$ for all but finitely many $n$. If $\alpha^{X}\left(d_{n}\right)<\alpha_{n}$ infinitely often then again $h^{X}$ would be a counterexample to the indiscernibility of $d_{n} \in \mathscr{D}\left(\alpha_{n}\right)$ in $V$, and hence there would be such a counterexample in $X$. Thus for infinitely many $n$ we have $\alpha_{n}=\alpha^{X}\left(d_{n}\right)$ and hence $d_{n} \in \mathscr{C}^{X}\left(\alpha_{n}\right)$, contradicting the definition of $\delta_{n}$ and $\nu_{n}$.

Proof of Theorem 1.1(i) assuming Lemma 4.3. Suppose that $X \prec H_{\kappa^{++}}$is a set as used in the covering lemma. Let $m=m^{X}$ be the covering mouse, let $h=h^{m^{X}} \cap \kappa^{2}$, and let $\rho$ be large enough that $X \cap \kappa \subset h^{\prime \prime}\left(\rho ; \mathscr{C}^{X}\right)$. We will assume that Corollary 2.8 holds for every ordinal $c$ in $\mathscr{C}^{X}$, increasing $\rho$ if necessary to be larger than any of the finitely many exceptions.

We will find an ordinal $\delta<\kappa$ such that $X \subset Y=Y^{\delta, h}=h^{\prime \prime}\left(\delta ; \mathscr{C}^{h}\right)$. Since there are only $\kappa^{+}$functions $h: \kappa \rightarrow \kappa$ in $K(\mathscr{F})$ and only $\kappa$ ordinals $\delta<\kappa$ there are only $\kappa^{+}$sets $Y^{\delta, h}$. Since each set $Y^{\delta, h}$ has cardinality $|\delta|<\kappa$ there are fewer than $\kappa$ subsets of $Y^{\delta, h}$. Thus there are only $\kappa^{+}$subsets of $\kappa$ of cardinality less then $\kappa$ and hence $2^{\kappa}=\kappa^{+}$.

Let $\delta$ be any ordinal in the interval $\rho<\delta<\kappa$ such that $\mathscr{C}^{X}(\alpha) \backslash \delta=$ $\mathscr{C}^{h}(\alpha) \backslash \delta$ for all $\alpha \in h^{\text {“c }}\left(\delta ; \mathscr{C}^{h}\right) \cap X$. We can always find such a $\delta$ since Lemma 4.3 asserts that for sufficiently large $\delta<\kappa$ we have $\mathscr{C}^{X}(\alpha) \backslash \delta \subset \mathscr{C}^{h}(\alpha)$ for all $\alpha \in h^{\prime \prime}\left(\delta ; \mathscr{C}^{h}\right)$, while Lemma 4.4 asserts that for sufficiently large $\delta<\kappa$ we have $\mathscr{C}^{X}(\alpha) \backslash \delta \supset \mathscr{C}^{h}(\alpha)$ for all $\alpha \in X$.

Now suppose $\nu \in(\kappa \cap X)$. We will show by induction on $\nu$ that $\nu \in Y=$ $h^{\prime \prime}\left(\delta ; \mathscr{C}^{h}\right)$. If $\nu<\delta$ then certainly $\nu \in Y$. If $\nu$ is not a full cardinal then either $\nu \in \sigma^{\prime \prime}\left((X \cap \nu)^{2}\right)$ or $\nu \in \tau^{\prime \prime}\left((X \cap \nu) \times[X \cap \nu]^{\omega}\right)$ and since $X \cap \nu \subset Y$ by the induction hypothesis $\nu$ is in $Y$ by Definition 4.2(ii) above. If $\nu$ is a full cardinal, but not an indiscernible in $\mathscr{C}^{X}$, then $\nu \in h^{X “}(\nu \cap X) \cap \kappa=h^{\prime \prime}(\nu \cap X) \subset$ $Y$, since $Y$ is closed under $h$. Thus we can assume that $\nu$ is an indiscernible in $\mathscr{C}^{X}$, so that $\nu=s^{\mathscr{C}^{X}}(\alpha, \beta, \gamma)$ for some $\gamma \in X \cap \nu$, some $\alpha \in h^{X “}(\nu \cap X)$, and some $\beta<\beta_{0}$. Then $\gamma \in Y$ by the induction hypothesis. The set $Y$ is closed under $h^{X}$ since it is closed under $h$. Hence $\alpha$ and $\beta$ are in $Y$ and by the choice of $\delta$ we have $\nu=s^{\mathscr{C}^{X}}(\alpha, \beta, \gamma)=s^{\mathscr{C}^{h}}(\alpha, \beta, \gamma) \in Y$.

This completes the proof of Theorem 1.1(i) from Lemma 4.3. The proof of Lemma 4.3 will be by induction on the domain $\eta$ of the function $h$. The next proposition will be needed for the induction step.

4.5 Proposition. Suppose that $h \in \mathscr{H}$ and $\mathscr{C}^{f}$ has been already defined as required by Lemma 4.3 for all functions $f \in \mathscr{H}$ with domain $(f) \leq \eta=$ domain $(h)$. Assume further that $X \prec H_{\kappa^{++}}$, the sequence $\left(\mathscr{C}^{f}: f \in \mathscr{H}\right.$ and domain $(f) \leq \eta)$ is a member of $X$, and $\rho<\eta$. Then there is a system $\mathscr{C} \in X$ such that $\mathscr{C}\left\lceil X=\mathscr{C}^{X}\left\lceil\left(h^{\prime \prime}\left(\rho ; \mathscr{C}^{X}\right) \cap((\eta+1) \backslash \rho)\right)\right.\right.$.

Proof. The proof is by induction on $\eta$. If $h^{\prime \prime}\left(\rho ; \mathscr{C}^{X}\right)$ is bounded in $\eta$ then the proposition follows immediately from the induction hypothesis, so we can assume that $h^{\prime \prime}\left(\rho ; \mathscr{C}^{X}\right)$ is unbounded in $\eta$.

Now Lemma 4.4 implies that there is $\delta_{0}<\eta$ such that $\mathscr{C}^{X}(\gamma) \supset \mathscr{C}^{h}(\gamma) \backslash \delta_{0}$ for all $\gamma \in X$, and the hypothesis to this proposition implies that there is $\delta_{1}$ such that $\mathscr{C}^{X}(\gamma) \backslash \delta_{1} \subset \mathscr{C}^{h}(\gamma)$ for all $\gamma \in h^{\prime \prime}\left(\zeta ; \mathscr{C}^{X}\right)$. Let $\delta=\max \left(\delta_{0}, \delta_{1}\right)$. 
Let $\vec{c}=\left\{c \leq \delta: \exists \alpha>\delta c \in \mathscr{C}^{X}(\alpha)\right.$ and $\left.\forall \alpha \leq \delta c \notin \mathscr{C}^{X}(\alpha)\right\}$. Then $\vec{c}$ is finite by Definition 2.5(ii)(c). Define $h^{\vec{c}} \in \mathscr{H}$ with domain $(h)=\delta$ by setting $h^{\vec{c}}(i)=c_{i}$ for $i<\operatorname{len}(\vec{c})$ and $h^{\vec{c}}(\operatorname{len}(\vec{c})+\nu)=h(\nu)$ otherwise. The induction hypothesis implies that there is a system $\mathscr{C}_{0} \in X$ of indiscernibles such that $\mathscr{C}_{0} \mid X=\mathscr{C}^{X} \uparrow\left(h^{\vec{c}^{\star c}}\left(\rho, \mathscr{C}^{X}\right) \cap(\delta+1 \backslash \rho)\right)$. Now define $\mathscr{C}_{1}$ by using $\vec{c}=\left(c_{i}: i<k\right)$ to combine $\mathscr{C}_{0}$ and $\mathscr{C}^{h} \uparrow(\eta \backslash \delta)$. That is,

$$
\mathscr{C}_{1}(\nu)=\left\{\begin{array}{l}
\mathscr{C}_{0}(\nu) \quad \text { if } \rho<\nu \leq \delta, \\
\mathscr{C}^{h}(\nu) \cup \cup\left\{\mathscr{C}_{0}\left(c_{i}\right) \cup\left\{c_{i}\right\}: c_{i} \in \mathscr{C}^{X}(\nu)\right\} \quad \text { if } \delta<\nu \leq \eta .
\end{array}\right.
$$

The system $\mathscr{C}=\mathscr{C}_{1} \mid h^{\prime \prime}\left(\rho ; \mathscr{C}_{1}\right)$ is the required system.

Proof of Lemma 4.3. We will construct the systems $\mathscr{C}^{h}$ by induction over $\eta=$ domain $(h)$. We assume as an induction hypothesis that $\mathscr{C}^{f}$ has been defined for all $f$ with domain $(f)<\eta$. If $\eta$ is regular then $h^{\text {“ }}(\rho ; \mathscr{C})$ cannot be cofinal in $\eta$, so we can define $\mathscr{C}^{h}(\nu)=\varnothing$ for all $\nu$. Now assume that $\eta$ is singular. If $\eta$ is singular in $K(\mathscr{F})$ then set $\lambda=\operatorname{cf}^{K(\mathscr{F})}(\eta)$, and otherwise let $\lambda=\operatorname{cf}(\eta)$. Take $X \prec H_{\eta^{++}}$so that $X$ is cofinal in $\eta$, the sequence $\left(\mathscr{C}^{f}: \operatorname{domain}(f)<\right.$ $\alpha)$ is in $X$, and $\beta_{0} \cup\left\{p, \beta_{0}, \lambda\right\} \subset X$. If $\eta$ is singular in $K(\mathscr{F})$ then let $\left(\gamma_{l}: l<\lambda\right) \in K(\mathscr{F}) \cap X$ be a closed unbounded subset of $\eta$. Otherwise $\mathscr{C}^{X}(\eta)$ is unbounded in $\eta$, so let $\left(\gamma_{l}: l<\lambda\right)$ be a $\omega$-closed, unbounded subset of $\mathscr{C}^{X}(\eta)$. For each $l<\lambda$ let $h_{l}=h \uparrow \gamma_{l+1}$ and use Proposition 0.0 to pick $\mathscr{C}^{l} \in X$ so that $\mathscr{C}^{\top} \uparrow X=\mathscr{C}^{X} \uparrow\left(h_{l}\left(\gamma_{l} ; \mathscr{C}^{X}\right) \cap\left(\gamma_{l+1}+1 \backslash \gamma_{l}\right)\right)$. Define $\mathscr{C}^{h}(\nu)=\mathscr{C}^{l}(\nu)$ for $\gamma_{l}<\nu \leq \gamma_{l+1}$, and if $\eta$ is measurable in $K(\mathscr{F})$ then set $\mathscr{C}^{h}(\eta)=\left\{\gamma_{l}: l<\lambda\right\} \cup \bigcup_{l} \mathscr{C}^{l}\left(\gamma_{l+1}\right)$.

The proof that $\mathscr{C}^{h}$ is a system of indiscernibles is just like that in Theorem 4.1: if it is not, then there is a countable set $I \subset \lambda$ such that $\mathscr{C}^{I}=$ $\mathscr{C}^{h} \backslash \bigcup_{t \in I}\left(\gamma_{t+1}+1 \backslash \gamma_{l}\right)$ is not a system of indiscernibles. But $\mathscr{C}^{I}$ is a member of $X$ so it is true in $X$ that $\mathscr{C}^{I}$ is not a system of indiscernibles, which is impossible since $\mathscr{C}^{I} \mid X$ is the restriction of $\mathscr{C}^{X}$ to the set $\bigcup_{l \in I}\left(\gamma_{t+1}+1 \backslash \gamma_{l}\right)$. Similarly if $\mathscr{C}^{h}$ does not satisfy the maximality property of lemma 4.3 then there must be another system $\mathscr{C}$ of indiscernibles and a countable set $I \subset \lambda$ such that for each $l \in I$ there is $\nu_{l} \in h^{\prime \prime}\left(\gamma_{l} ; \mathscr{C}\right) \cap\left(\gamma_{i}, \gamma_{l+1}+1\right]$ such that $\mathscr{C}\left(\nu_{l}\right) \backslash \gamma_{l}$ is not contained in $\mathscr{C}^{I}\left(\nu_{l}\right)$. But then there must be such a system $\mathscr{C}$ and sequence $\nu$ which is a member of $X$, so that except for finitely many $l$ we must have $\mathscr{C}\left(\nu_{l}\right) \subset \mathscr{C}^{X}\left(\nu_{l}\right)=\mathscr{C}^{h}\left(\nu_{l}\right)$, a contradiction.

I would like to thank the referee for a careful reading of this paper and many helpful suggestions.

\section{REFERENCES}

[D] A. Dodd, The core model, London Math. Soc. Lecture Notes 61, Cambridge Univ. Press, Cambridge, 1982.

[G] M. Gitik, The negation of the singular cardinal hypothesis from $o(\kappa)=\kappa^{++}$, Ann. Pure Appl. Logic 43 (1989), 209-234.

[G?] _ The strength of the failure of the singular carrdinal hypothesis, Ann. Pure Appl. Logic (to appear).

[G??] _ On measurable cardinals violating the continuum hypothesis, preprint. 
[KM] A. Kanamori and M. Magidor, The evolution of large cardinal axioms in set theory, Higher Set Theory, Gert Muller and Dana Scott, Eds., Lecture Notes in Math., vol. 669, SpringerVerlag, Berlin, 1978, pp. 99-276.

[Mc] McDermitt, Iterated forcing and coherent sequences, Thesis, University of Oxford.

[Mi74] W. J. Mitchell, Sets constructible from sequences of ultrafilters, J. Symbolic Logic 39 (1974), 57-66.

[Mi83] _ Sets constructed from sequences of measures: Revisited, J. Symbolic Logic 48 (1983), 600-607.

[Mi84a] _ Indiscernibles, skies and ultrafilters, Contemp. Math. 31 (1984), 161-182.

[Mi84b] _ The core model for sequences of measures, I, Math. Proc. Cambridge Philos. Soc. 95 (1984), 41-58.

[Mi?] _ The core model for sequences of measures, II, Math. Proc. Cambridge Philos. Soc. (submitted).

[Mi87] _ Applications of the core model for sequences of measures, Trans. Amer. Math. Soc. 299 (1987), 41-58.

[W] H. Woodin, (Private communication).

[Pr] K. Prikry, Changing measurable into accessible cardinals, Dissertationes Math. (Rozprawy Math.) 68 (1971), 359-378.

Department of Mathematics, University of Florida, Gainesville, Florida 32611

E-mail address: mitchell@math.ufl.edu 